Article

\title{
Synthesis and Antimicrobial Activity of Some New Substituted Quinoxalines
}

\author{
Mohamed A. El-Atawy ${ }^{1,2}, *$ (D) Ezzat A. Hamed ${ }^{2}$, Mahjoba Alhadi ${ }^{2}$ and Alaa Z. Omar ${ }^{2}$ \\ 1 Chemistry Department, Faculty of Science, Taibah University, Yanbu 46423, Saudi Arabia \\ 2 Chemistry Department, Faculty of Science, Alexandria University, P.O. 426 Ibrahemia, \\ Alexandria 21321, Egypt; ezzat.awad@alexu.edu.eg (E.A.H.); mahjoba.alhadi@yahoo.com (M.A.); \\ Alaazaki@alexu.edu.eg (A.Z.O.) \\ * Correspondence: mohamed.elatawi@alexu.edu.eg; Tel.: +966-569-191-532
}

Received: 12 September 2019; Accepted: 12 November 2019; Published: 19 November 2019

check for updates

\begin{abstract}
A number of new symmetrically and asymmetrically 2,3-disubstituted quinoxalines were synthesized through functionalization of 2,3-dichloroquinoxaline (2,3-DCQ) with a variety of sulfur and/or nitrogen nucleophiles. The structures of the obtained compounds were established based on their spectral data and elemental analysis. The antimicrobial activity for the prepared compounds was investigated against four bacterial species and two fungal strains. The symmetrically disubstituted quinoxalines 2, 3, 4, and 5 displayed the most significant antibacterial activity, while compounds $6 \mathbf{a}, \mathbf{6 b}$, and the pentacyclic compound 10 showed considerable antifungal activity. Furthermore, compounds $\mathbf{3} \mathbf{f}, \mathbf{6} \mathbf{b}$ showed broad antimicrobial spectrum against most of the tested strains.
\end{abstract}

Keywords: quinoxalines; antimicrobial; thioether; thioglycolic acid and pentacyclic compounds

\section{Introduction}

The quinoxaline nucleus is one of the most important heterocyclic scaffolds. Quinoxalines exist in several different biologically active compounds including some antibiotics such as levomycin, actinoleutin and echinomycin which are known to be active against several transplantable tumors [1]. Beside that they have different applications in many fields such as agricultural [2], fluorescent materials [3], dyes [4], electroluminescent materials [5], organic semiconductors [6], organic light emitting devices [7], and medicinal chemistry. Various synthetic quinoxalines exhibit miscellaneous therapeutic applications in medicinal research such as anticancer [8-10], anti-inflammatory [9,11], antiviral [12,13], antidiabetic [14,15], antidepressant [16], anthelmintic [2], antituberculosis [17], antibacterial $[18,19]$, and antiprotozoal $[20,21]$. Two clinical examples for drugs containing quinoxaline are illustrated in Figure 1. Namely, chlorosulfaquinoxaline [22] and $R-(+)-X K 469$ [23]. The former drug has immunosuppressive activity and potential antineoplastic activity [24] the second one is an anticancer drug that has activity against solid tumors [25]. Furthermore, quinoxalines are used in the agricultural field as herbicides, insecticides, and fungicides [2]. Thus, due to this diversity of useful applications for quinoxalines, scientists pay much attention to the classical synthetic methods, their modification, and for new methods for synthesizing quinoxalines to ensure the availability of more functionalized quinoxalines.

Organosulfur compounds are well defined as organic molecules having one or more carbon-sulfur bonds. These compounds constitute very diverse chemical structures and display several bioactive properties as well as antimicrobial; the antimicrobial activity of some organosulfur compounds has been reported against a wide spectrum of fungi, bacteria, and viruses [26]. Some of these organosulfur compounds are also used in the treatment of various types of tumors caused by Helicobacter pylori along with gastric ulcers. Moreover, existence of sulfur and nitrogen usually improve the efficiency 
of some compounds for the treatment of several types of fungal, bacterial, and different pathogen causing diseases [27-29]. Recently, antimicrobial activity of trisubstituted quinoxaline derivatives has been reported [19]. It was observed that 4-triflouromethylanilino, 4-hydroxyanilino or phenylthio groups at positions 2 and/or 3 of quinoxaline ring are responsible for good to moderate antibacterial activity. However, pipridino or morpholino groups at these positions reduced the antibacterial activity. Furthermore, most of tested quinoxalines showed moderate to weak antifungal activity. Inspired and motivated by these findings and by the importance of sulfur and nitrogen containing compounds, we tried to improve the antimicrobial activity of quinoxaline by its functionalization with sulfur and/or nitrogen. In order to identify new candidates that may be of value in designing new, selective, and less toxic antimicrobial agents. Herein we report our results on functionalization of quinoxaline with a variety of sulfur and nitrogen nucleophiles using classical methods and testing the products for their biological activities.<smiles>Nc1ccc(S(=O)(=O)Nc2cnc3c(Cl)cccc3n2)cc1</smiles>

Chlorosulfaquinoxaline<smiles>C[C@H](Oc1ccc(Oc2cnc3ccc(Cl)cc3n2)cc1)C(=O)O</smiles>

$R-(+)-X K 469$

Figure 1. Examples for drugs containing quinoxaline moiety.

\section{Results and Discussion}

\subsection{Chemistry}

Among the different methods for synthesizing symmetrically 2,3-disubstituted quinoxalines, there are two common methods widely used. The first one includes cyclocondensation reaction between symmetric alpha diketone such as benzil derivatives and o-phenylenediamine [30-32]. In general, this procedure needs use of a strong acid catalyst, high temperature, and long reaction times. Moreover, the main drawback of this method is its scope, which is limited to the synthesis of dialkyl or diaryl quinoxaline. The second method involves nucleophilic substitution reaction between 2,3-DCQ and nucleophiles [19]. This method has broader scope due to the availability of the starting materials and the ease of their preparation. In the current work, a number of symmetrically 2,3disubstituted quinoxalines have been prepared through simple nucleophilic displacement on 2,3-DCQ using either sulfur or nitrogen nucleophiles affording products $\mathbf{2 a - e}$ and $\mathbf{3 a}-\mathbf{g}$, respectively as shown in Scheme 1. The yields of the reaction varied from good to excellent yield. The starting compound 1 was prepared by cyclocondensation reaction between $o$-phenylenediamine and oxalic acid in strong acidic medium, to give quinoxaline-2,3-dione [33]. Which, on subsequent chlorination with thionyl chloride or phosphorus oxychloride afforded the desired starting 2,3-DCQ 1. The S-aryl or NH-aryl groups of the products $\mathbf{2 a - e}$ and $\mathbf{3 a - g}$, respectively, bear a variety of substituents including halogens $(\mathrm{F}, \mathrm{Br}$, and $\mathrm{Cl})$, alkyl and alkoxy groups. Infrared spectrum of products $3 \mathrm{a}-\mathrm{g}$ showed bands in the region $3200-3300 \mathrm{~cm}^{-1}$ that can be ascribed to the absorption characteristic for N-H stretching. Moreover, proton NMR (DMSO- $d_{6}$ ) of 3a-g revealed broad $\mathrm{D}_{2} \mathrm{O}$-exchangeable singlets at $\delta 11.93,9.63,9.16,8.92$, $9.85,8.84$, and $8.87 \mathrm{ppm}$, respectively, due to the existence of $\mathrm{NH}$ protons. The infrared spectrum of products $2 \mathbf{e}, \mathbf{3 d}-\mathbf{g}$, and 4 exhibited absorption bands at $2916-2999 \mathrm{~cm}^{-1}$ corresponding to the aliphatic C-H stretching. The methyl groups of compounds $\mathbf{2 e}, \mathbf{3 d}, \mathbf{3 e}, \mathbf{3 g}$, and $\mathbf{3 f}$ were displayed in both proton and ${ }^{13} \mathrm{C}$-NMR spectrum. Accordingly, methyl protons of $\mathbf{2 e}, 3 \mathbf{d}$ revealed peaks in ${ }^{1} \mathrm{H}-\mathrm{NMR}$ at $\delta 2.42,2.30 \mathrm{ppm}$, respectively. Additionally, ${ }^{13} \mathrm{C}-\mathrm{NMR}$ spectrum of $\mathbf{2 e}, \mathbf{3 d}$ exhibited signals at $\delta$ $21.28,20.51 \mathrm{ppm}$ respective to their methyl carbon. The methyl groups of $3 \mathbf{e}$ showed two singlets in ${ }^{1} \mathrm{H}-\mathrm{NMR}$ at 2.23 and $2.26 \mathrm{ppm}$ and two signals in ${ }^{13} \mathrm{C}-\mathrm{NMR}$ at 18.89 and $19.72 \mathrm{ppm}$. However, the methoxy groups of $3 \mathrm{~g}$ were observed in ${ }^{1} \mathrm{H}-\mathrm{NMR}$ spectrum as more deshielded singlets at 3.76 and 
$3.81 \mathrm{ppm}$ and in ${ }^{13} \mathrm{C}-\mathrm{NMR}$ spectrum as two signals at 55.78 and $55.40 \mathrm{ppm} .{ }^{1} \mathrm{H}-\mathrm{NMR}$ spectrum of $3 \mathrm{f}$ exhibited a singlet at $3.76 \mathrm{ppm}$ attributable to methoxy protons. Furthermore, methoxy carbon of $\mathbf{3 f}$ appeared as a signal at $55.22 \mathrm{ppm}$ in ${ }^{13} \mathrm{C}-\mathrm{NMR}$. The formation of compound 4 has been confirmed by the two singlets that appeared in ${ }^{1} \mathrm{H}-\mathrm{NMR}$ at $11.50,4.17 \mathrm{ppm}$ due to $\mathrm{COOH}$ and $\mathrm{CH}_{2}$, respectively. Additionally, ${ }^{13} \mathrm{C}-\mathrm{NMR}$ of 4 showed two signals at 169.53 and $32.49 \mathrm{ppm}$ corresponding to the carbons of the carboxymethyl moiety $\left(\mathrm{CH}_{2} \mathrm{COOH}\right)$. Furthermore, Conformation of the symmetrical structures of the products (2a-e, $\mathbf{3 a - g}$, and $\mathbf{4})$ has been achieved mainly through NMR spectroscopic data. As it is well known that symmetry-equivalent atoms must absorb at the same chemical shift. Accordingly, due to the molecular symmetry of the products $(2 \mathbf{a}-\mathbf{e}, 3 \mathbf{a}-\mathbf{g}$, and 4$)$ their quinoxaline moiety showed up two peaks in proton NMR and two quaternary carbons in ${ }^{13} \mathrm{C}-\mathrm{APT}-\mathrm{NMR}$. As a prototype, the ${ }^{13} \mathrm{C}$-APT-NMR of compound $2 \mathbf{a}$ in DMSO- $d_{6}$ revealed the presence of three quaternary carbons at $128.70,140.37$, and $153.73 \mathrm{ppm}$. The last two peaks (153.73 and $140.37 \mathrm{ppm}$ ) were assigned to the quaternary carbons of the quinoxaline moiety of $\mathbf{2 a}$, while the peak that appeared at $128.70 \mathrm{ppm}$ was referred to as the ipso quaternary carbon of the thiophenyl ring. Nevertheless, the different environments for the protons or carbons of non-symmetrical structures $(5,6 \mathbf{a}, \mathbf{b}, 7, \mathbf{8}, \mathbf{9 a}-\mathbf{f}$, and 10) led to appearance of four protons and four quaternary carbons corresponding to their quinoxaline moiety as illustrated in Figure 2.

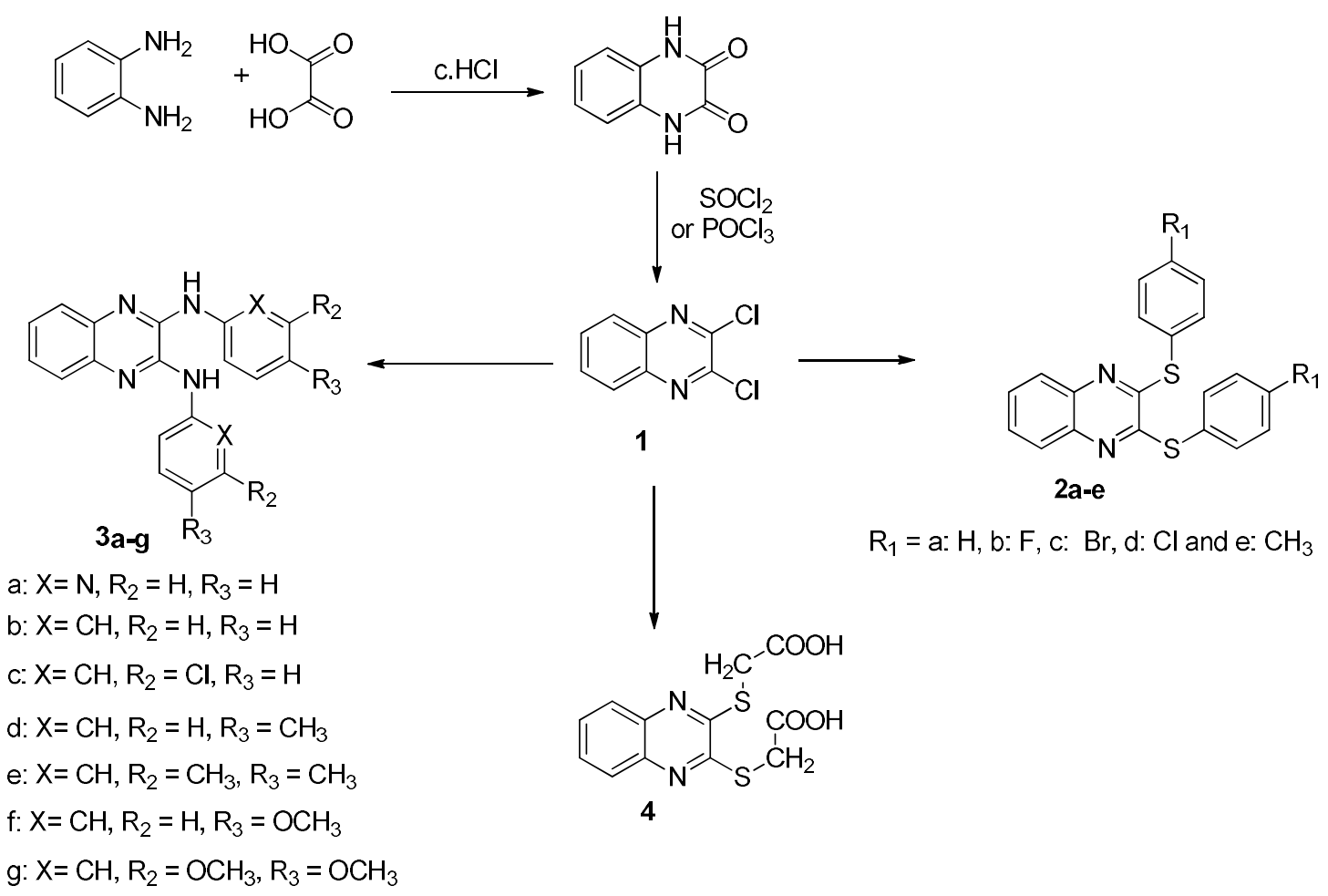

Scheme 1. Synthesis of sym. 2,3-disubstituted quinoxaline.

As an extension to this study, a variety of asymmetrically 2,3-disubstituted quinoxaline containing sulfur and/or nitrogen substituents have been synthesized (Scheme 2). One of the challenges of this work was how to find a simple and efficient method for the synthesis of asym. disubstituted quinoxalines. In a previously reported method, vinylic substitution reaction occurred between 1,1-dimethylsulfanyl-2-nitroethene and appropriate aniline derivative to afford (N-(1-(methylthio)-2-nitrovinyl)aniline which on subsequent reductive cyclization gave 2-chloro-3-(methylthio)quinoxaline [34] as a precursor for transformation into asym. disubstituted quinoxalines. However, a more convenient approach for the synthesis of these quinoxaline derivatives is the substitution of one of the chlorines at the 2- or 3-position of 2,3-DCQ by nucleophiles. The reaction was conducted at room temperature with continuous stirring to avoid occurrence of double substitution 
on 1. Consequently, 2,3-DCQ was allowed to react with 4-chloroaniline, thiophenol, thiosalicylic acid, and hydrazine to afford compounds $5,6 \mathbf{6}, \mathbf{6 b}$, and 7, respectively. These products contain chlorinated quinoxaline as analogue to several biologically active quinoxalines [35]. Reaction of either 6a with hydrazine or reaction of 7 with thiophenol gave the same compound $\mathbf{8}$, which on subsequent condensation with different aromatic aldehyde afforded hydrazones 9a-f. Furthermore, 2,3-DCQ was reacted with benzimidazole-2-thiol as a binucleophile affording the pentacyclic compound 10. Thus, we synthesized 19 new 2,3-disubstituted quinoxaline derivatives (2b-e; 3a; 3e; 3f; $\mathbf{4} ; \mathbf{5} ; \mathbf{6 a} \mathbf{6} \mathbf{b} ; \mathbf{8} ; \mathbf{9 a}-\mathbf{f})$ and seven previously reported compounds (1; 2a [36]; 3b; 3c [37]; 3d; 3g, 7) using a simple and efficient synesthetic method with the goal of discovering their antimicrobial activity.

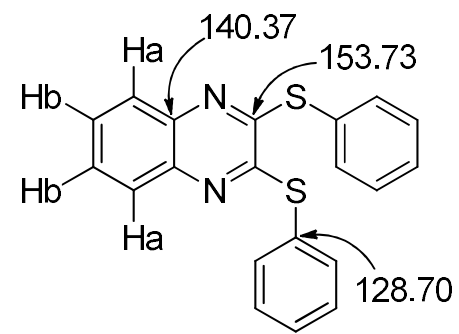

$2 a$

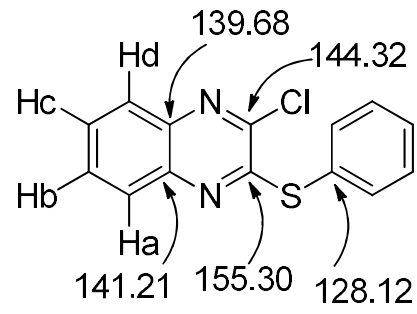

$6 b$

Figure 2. Quinoxaline moiety of $\mathbf{2 a}$ showed only two protons and two quaternary carbon in NMR. $\mathbf{6 b}$ showed four different types of protons and four different quaternary carbons, which correspond to its quinoxaline moiety.

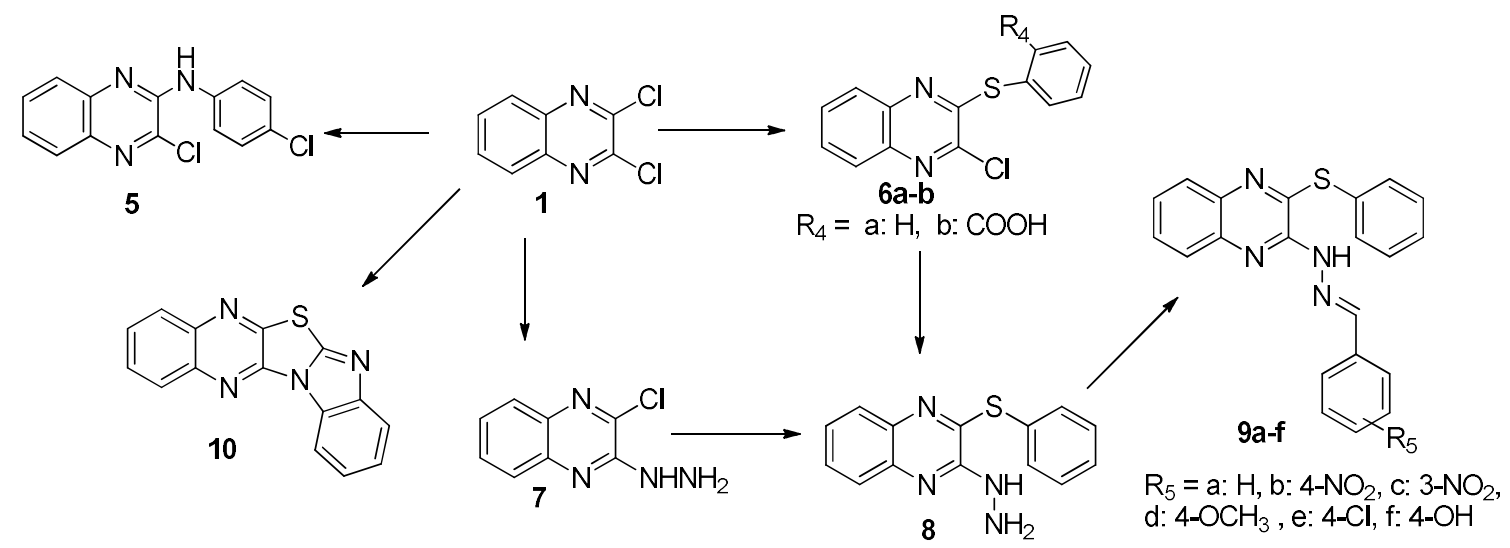

Scheme 2. Synthesis of asym. 2,3-disubstituted quinoxaline.

Previously, hydrazones derived from quinoxaline have been reported for their antimicrobial and anti-inflammatory effects [9,38-40]. That enhanced us to design quinoxaline derivatives 9a-f which contain both thioether and arylhydrazones functionalities at 2- and 3-positions of quinoxaline ring for testing their biological activity.

The newly prepared compounds 5-10 gave analytical and spectral data in agreement with their structures. For example, compounds 5, 7, 8, and 9a-f showed the characteristic infrared absorption bands for N-H stretching at 3300-3358 $\mathrm{cm}^{-1}$ in addition, proton NMR of those compounds showed $\mathrm{D}_{2} \mathrm{O}$-exchangeable broad singlets, which confirmed the existence of $\mathrm{NH}$ and/or $\mathrm{NH}_{2}$ groups. Condensation reaction of $\mathbf{8}$ with six different aromatic aldehydes led to formation of Schiff bases $\mathbf{9 a - f}$. Those Schiff bases showed a new singlet at $\delta 8.46-8.72$ ppm corresponding to azomethine proton. Furthermore, the azomethine carbon appeared in carbon NMR at $\delta 138-145$ ppm. 


\subsection{Biological Evaluation}

In vitro antimicrobial screening of the synthesized compounds $\mathbf{2 a - d , 3 a - g , ~ 4 , ~ 5 , ~ 6 a , b , ~ 8 , ~ a n d ~ 9 a - f ~ w a s ~}$ estimated against four bacterial species, namely Bacillus subtilis, Staphylococcus aureus as a Gram-positive bacterium. Moreover, Escherichia coli and Proteus vulgaris as a Gram-negative bacterium. In addition, the study also included two fungal strains, namely Candida albicans and Aspergillus flavus. Gentamycin and Ketoconazole were used as antibacterial and antifungal reference drugs, respectively. Dimethyl sulfoxide (DMSO) was used as solvent as well as negative reference. The activities of the tested compounds are summarized in Table 1. The data obtained allowed the following observations and conclusions: the negative reference, dimethyl sulfoxide, did not show any inhibition zone against all the tested strains. The antibacterial positive reference, Gentamycin, showed an average inhibition zone of 24.00, 26.00, 30.00, and $25.00 \mathrm{~mm}$ against the strains of Staphylococcus aureus; Bacillus subtilis; Escherichia coli and Proteus vulgaris, respectively. The antifungal positive reference, Ketoconazole, showed an average inhibition zone of $20.00 \mathrm{~mm}, 16.00 \mathrm{~mm}$ against the strains of, Candida albicans and Aspergillus flavus, respectively. The tested compounds showed significant antibacterial activity against the strains of Staphylococcus aureus, Bacillus subtilis, and Escherichia coli relative to the strain of Proteus vulgaris. Furthermore, among all the tested compounds, only compounds $\mathbf{6 a}, \mathbf{6} \mathbf{b}$ and the pentacyclic compound $\mathbf{1 0}$ have considerable antifungal activity. Compounds $\mathbf{3 f}, \mathbf{6 b}$ showed broad antimicrobial spectrum against most of the tested strains. The symmetrically disubstituted quinoxalines 2, 3, 4, and 5 presented the most significant antibacterial activity. However, the asymmetrically substituted quinoxalines 8, 9, and 10 showed reduced antibacterial activity. The hydrazine $\mathbf{8}$ revealed a slightly lower antimicrobial activity than its corresponding hydrazones $\mathbf{9 a - g}$.

Table 1. Antimicrobial activities of compounds 2-6 and 8-10.

\begin{tabular}{|c|c|c|c|c|c|c|}
\hline \multirow[b]{3}{*}{ Compounds } & \multicolumn{4}{|c|}{ Antibacterial Activity Zone of Inhibition (mm) } & \multicolumn{2}{|c|}{ Antifungal Activity Zone of Inhibition (mm) } \\
\hline & \multicolumn{2}{|c|}{$\mathrm{G}+$ bacteria } & \multicolumn{2}{|c|}{$\mathbf{G}-$ bacteria } & \multirow[b]{2}{*}{ C. albicans } & \multirow[b]{2}{*}{ A. flavus } \\
\hline & S. aureus & B. subtilis & E. coli & P. vulgaris & & \\
\hline $2 a$ & 26 & 30 & 18 & 15 & 17 & 14 \\
\hline $2 b$ & 22 & 19 & 25 & 39 & 16 & 12 \\
\hline $2 c$ & 25 & 20 & 27 & 31 & 13 & 15 \\
\hline $2 d$ & 28 & 44 & 34 & 11 & 11 & 5 \\
\hline $3 a$ & 13 & 12 & 14 & 24 & 19 & 14 \\
\hline $3 b$ & 19 & 28 & 39 & 23 & 18 & 9 \\
\hline $3 c$ & 35 & 41 & 37 & 15 & 18 & 16 \\
\hline $3 d$ & 16 & 30 & 32 & 27 & 11 & 19 \\
\hline $3 e$ & 26 & 24 & 27 & 16 & 18 & 11 \\
\hline $3 f$ & 30 & 29 & 30 & 28 & 17 & 14 \\
\hline $3 g$ & 37 & 29 & 28 & 16 & 17 & 15 \\
\hline 4 & 27 & 38 & 12 & 15 & 18 & 15 \\
\hline 5 & 22 & 37 & 37 & 24 & 18 & 12 \\
\hline $6 a$ & 22 & 35 & 24 & 19 & 25 & 17 \\
\hline $6 b$ & 29 & 31 & 35 & 23 & 22 & 11 \\
\hline 8 & 15 & 9 & 9 & 11 & 7 & 9 \\
\hline $9 a$ & 22 & 18 & 25 & 23 & 10 & 9 \\
\hline $9 b$ & 17 & 13 & 18 & 20 & 11 & 11 \\
\hline $9 c$ & 14 & 12 & 22 & 21 & 10 & 15 \\
\hline $9 d$ & 20 & 11 & 14 & 12 & 11 & 7 \\
\hline $9 e$ & 19 & 10 & 19 & 18 & 19 & 5 \\
\hline $9 f$ & 13 & 15 & 17 & 11 & 11 & 8 \\
\hline 10 & 13 & 26 & 24 & 15 & 34 & 27 \\
\hline Ketoconazole & - & - & - & - & 20 & 16 \\
\hline Gentamycin & 24 & 26 & 30 & 25 & - & - \\
\hline DMSO & 0 & 0 & 0 & 0 & 0 & 0 \\
\hline
\end{tabular}

S. aureus, Staphylococcus aureus (RCMB010010); B. subtilis, Bacillus subtilis (RCMB 015 (1) NRRL B-543); E. coli, Escherichia coli (RCMB 010052) ATCC 25955; P. vulgaris, Proteus vulgaris RCMB 004 (1) ATCC 13315; C. albicans, Candida albicans RCMB 005003 (1) ATCC 10231; A. flavus, Aspergillus flavus (RCMB 002002). 
The minimum inhibitory concentration (MIC) is the lowest concentration causing full inhibition of the tested microorganism's growth. MIC is a further test applied for selected compounds with good antimicrobial activity. The MIC were determined via the double dilution technique [41]. Six concentrations were prepared for each selected compound (8, 16, 32, 64, 128, and $256 \mu \mathrm{g} / \mathrm{mL}$ ). The bacteria were inoculated and incubated at $37{ }^{\circ} \mathrm{C}$ for $24 \mathrm{~h}$ in nutrient broth medium; however, the fungal strains were inoculated in malt extract broth and incubated for $48 \mathrm{~h}$. MIC values of the tested compounds for different microorganisms are given in Table 2. As summarized in Table 2, the pentacyclic compound 10 exhibited the highest antifungal activity against both Candida albicans and Aspergillus flavus with MIC $16 \mu \mathrm{g} / \mathrm{mL}$. On the other hand, the tested compounds $2 \mathrm{~d}$ and $3 \mathrm{c}$ showed the highest antibacterial activity against the Gram-negative bacterium Escherichia coli with MIC $8 \mu \mathrm{g} / \mathrm{mL}$. Moreover, Compounds 2d, 3c, 4, and 6a presented the highest antibacterial activity against the Gram-positive bacterium Bacillus subtilis with MIC $16 \mu \mathrm{g} / \mathrm{mL}$. Besides that, many of the tested compounds presented a good antibacterial activity comparable to Gentamycin (i.e., compounds $2 \mathrm{a}, \mathbf{2 c}, \mathbf{2 d}, \mathbf{3 c}, \mathbf{3 f}, \mathbf{3 g}$, 4, and 6b against Staphylococcus aureus; compounds $\mathbf{2 a}, \mathbf{3 d}, \mathbf{3 f}, \mathbf{3 g}, \mathbf{6 b}$, and $\mathbf{1 0}$ against Bacillus subtilis; compounds 2c and 3d against Proteus vulgaris).

Table 2. The minimal inhibitory concentration (MIC: $\mu \mathrm{g} / \mathrm{mL}$ ) of the tested compounds.

\begin{tabular}{ccccccc}
\hline Compounds & S. aureus & B. subtilis & E. coli & P. vulgaris & C.albicans & A. flavus \\
\hline $\mathbf{2 a}$ & 32 & 32 & 64 & 64 & 64 & 64 \\
$\mathbf{2 b}$ & 64 & 128 & 64 & 8 & 64 & 256 \\
$\mathbf{2 c}$ & 32 & 64 & 64 & 16 & 128 & 64 \\
$\mathbf{2 d}$ & 32 & 16 & 8 & 128 & 256 & $>256$ \\
$\mathbf{3 a}$ & $>256$ & $>256$ & $>256$ & 256 & 128 & 128 \\
$\mathbf{3 b}$ & 256 & 64 & 16 & 32 & 64 & $>256$ \\
$\mathbf{3 c}$ & 32 & 16 & 8 & 64 & 64 & 64 \\
$\mathbf{3 d}$ & 256 & 32 & 16 & 16 & $>256$ & 64 \\
$\mathbf{3 e}$ & 64 & 128 & 128 & 128 & 64 & 128 \\
$\mathbf{3 f}$ & 32 & 32 & 32 & 32 & 128 & 256 \\
$\mathbf{3 g}$ & 32 & 32 & 16 & 64 & 128 & 128 \\
$\mathbf{4}$ & 32 & 16 & $>256$ & $>256$ & 64 & 64 \\
$\mathbf{5}$ & 256 & 32 & 16 & 32 & 64 & 256 \\
$\mathbf{6 a}$ & 64 & 16 & 64 & 32 & 16 & 32 \\
$\mathbf{6 b}$ & 32 & 32 & 16 & 32 & 32 & $>256$ \\
$\mathbf{1 0}$ & 256 & 32 & 32 & 32 & 16 & 16 \\
Ketoconazole & - & - & - & - & 32 & 32 \\
Gentamycin & 32 & 32 & 16 & 16 & - & - \\
\hline
\end{tabular}

S. aureus, Staphylococcus aureus (RCMB010010); B. subtilis, Bacillus subtilis (RCMB 015 (1) NRRL B-543); E. coli, Escherichia coli (RCMB 010052) ATCC 25955; P. vulgaris, Proteus vulgaris RCMB 004 (1) ATCC 13315; C. albicans, Candida albicans RCMB 005003 (1) ATCC 10231; A. flavus, Aspergillus flavus (RCMB 002002).

\section{Experimental}

\subsection{Instruments and Apparatus}

Melting points were determined by MEL-TEMP II melting point apparatus in open glass capillaries and were uncorrected. The IR spectra were recorded as potassium bromide (KBr) discs on a Perkin-Elemer FT-IR (Fourier-Transform Infrared Spectroscopy), Faculty of Science, Alexandria University. The NMR spectra were carried out at ambient temperature $\left(\sim 25^{\circ} \mathrm{C}\right)$ on a (JEOL) $500 \mathrm{MHz}$ spectrophotometer using tetramethylsilane (TMS) as an internal standard, NMR Unit, Faculty of Science, Mansoura University. Elemental analyses were analyzed at the Micro analytical Unit, Faculty of Science, Cairo University. The biological evaluation was carried out in the Medical Mycology Laboratory of the Regional Center for Mycology and Biotechnology of Al-Azhar University, Cairo, Egypt. 


\subsection{Agar Disk-Diffusion Method}

The desired compounds were dissolved in DMSO (which has no inhibition activity) to obtain concentrations of $250 \mathrm{ppm}$ and soaked in filter paper disks of $5 \mathrm{~mm}$. The test was performed on medium potato dextrose agar (PDA) which contains an infusion of $200 \mathrm{~g}$ potatoes, $6 \mathrm{~g}$ dextrose, and $15 \mathrm{~g}$ agar. Uniform size filter paper disks (three disks per compound) were impregnated with equal volume $(10 \mu \mathrm{L})$ from the specific concentration of dissolved tested compounds and then carefully placed on the incubated agar surface. After incubation for $36 \mathrm{~h}$ at $27^{\circ} \mathrm{C}$ in the case of bacteria and for $48 \mathrm{~h}$ at $24^{\circ} \mathrm{C}$ in the case of fungi, inhibition of the organisms (evidenced by a clear zone surrounding each disk) was measured and used to calculate the mean of inhibition zones [42].

\subsection{Determination of MIC}

All the bacteria were incubated and activated at $37^{\circ} \mathrm{C}$ for $24 \mathrm{~h}$ inoculation into nutrient broth and the fungi were incubated in malt extract broth for $48 \mathrm{~h}$. The compounds were dissolved in DMSO and then diluted using cautiously adjusted Mueller-Hinton broth. Two-fold serial concentrations dilution method $(8,16,32,64,128$, and $256 \mu \mathrm{g} / \mathrm{mL})$ of some compounds were employed to determine the MIC values. In each case, triplicate tests were performed, and the average was taken as the final reading. The tubes were then inoculated with the test organisms, grown in their suitable broth at $37^{\circ} \mathrm{C}$ for $24 \mathrm{~h}$ for tested microorganisms $\left(1 \times 10^{8} \mathrm{CFU} / \mathrm{mL}\right.$ for bacteria and $1 \times 10^{6} \mathrm{CFU} / \mathrm{mL}$ of fungi), each $5 \mathrm{~mL}$ received $0.1 \mathrm{~mL}$ of the above inoculum and was incubated at $37^{\circ} \mathrm{C}$ for $24 \mathrm{~h}$. The lowest concentration showing no growth was taken as the minimum inhibitory concentration (MIC); shown in Table 2.

\subsection{Synthesis of Quinoxaline-2,3-dione}

$o$-Phenylenediamine (27.9 g, $0.25 \mathrm{~mol})$, oxalic acid $(32.5 \mathrm{~g}, 0.36 \mathrm{~mol})$, and $4 \mathrm{~N} \mathrm{HCl}(150 \mathrm{~mL})$ were refluxed for $4 \mathrm{~h}$ then cooled. The solid separated was filtered, washed, and used without further purification, yield (86\%), m.p. $>300{ }^{\circ} \mathrm{C}$ [lit. $\left.>300{ }^{\circ} \mathrm{C}\right]$ [43], ${ }^{1} \mathrm{H}-\mathrm{NMR}\left(500 \mathrm{MHz}, \mathrm{DMSO}-d_{6}, 300 \mathrm{~K}\right)$, $\delta 11.91\left(\mathrm{~s}, 2 \mathrm{H}, 2 \mathrm{NH}, \mathrm{D}_{2} \mathrm{O}\right.$ exchangeable), $7.11\left(\mathrm{dd}, 2 \mathrm{H}, J=6.1,3.3 \mathrm{~Hz}, \mathrm{H}_{6}\right.$ and $\left.\mathrm{H}_{7}\right)$ and $7.07(\mathrm{dd}, 2 \mathrm{H}$, $J=6.0,3.3 \mathrm{~Hz}, \mathrm{H}_{5}$ and $\left.\mathrm{H}_{8}\right)$ ppm.

\subsection{Synthesis of 2,3-DCQ (1)}

Method A: Equimolar quantities of quinoxaline-2,3-dione (16.013 g, $0.1 \mathrm{~mol})$ and phosphorus oxychloride ( $15.333 \mathrm{~g}, 0.1 \mathrm{~mol}$ ) were mixed and the resulting mixture was refluxed on a water bath for $2 \mathrm{~h}$. The reaction mixture was then cooled to room temperature and the resulting precipitate was isolated by filtration, washed with water, then crystalized from rectified spirit as colorless crystals, $69 \%$ yield; m.p. $143-145^{\circ} \mathrm{C}$ [lit. $\left.148-150{ }^{\circ} \mathrm{C}\right]$ [44].

Method B: A mixture of quinoxaline-2,3-dione (3.08 g, $0.019 \mathrm{~mol})$ in 1,2-dichloroethane $(25 \mathrm{~mL})$ and thionyl chloride $(4 \mathrm{~mL}, 0.055 \mathrm{~mol})$ dissolved in few drops of dimethylformamide was heated on water bath for $4 \mathrm{~h}$. The reaction mixture was then cooled to room temperature and the resulting precipitate was isolated by filtration, washed with water, then crystalized from rectified spirit as colorless crystals, $82 \%$ yield; m.p. $143-145{ }^{\circ} \mathrm{C} .{ }^{1} \mathrm{H}-\mathrm{NMR}\left(500 \mathrm{MHz}: \mathrm{CDCl}_{3}\right), \delta 8.01(\mathrm{dd}, 2 \mathrm{H}, J=6.4$, $3.5 \mathrm{~Hz}, \mathrm{H}_{6}$ and $\left.\mathrm{H}_{7}\right)$ and $7.79\left(\mathrm{dd}, 2 \mathrm{H}, J=6.4,3.4 \mathrm{~Hz}, \mathrm{H}_{5}\right.$ and $\left.\mathrm{H}_{8}\right) \mathrm{ppm}$.

\subsection{Synthesis of 2,3-bis(arylthio)quinoxaline (2a-e)}

In a typical experiment a solution of 2,3-DCQ $1(1 \mathrm{~g}, 0.005 \mathrm{~mol})$ and $0.015 \mathrm{~mol}$ of appropriate thiol (namely, $4 \mathrm{~mL}$ thiophenol, $1.28 \mathrm{~mL} p$-fluorothiophenol, $2.85 \mathrm{~g} p$-bromothiophenol, $2.16 \mathrm{~g}$ $p$-chlorothiophenol or $1.86 \mathrm{~g} p$-methylthiophenol) and triethylamine $(3.03 \mathrm{~g}, 0.03 \mathrm{~mol})$ in $30 \mathrm{~mL}$ methanol was refluxed for $2 \mathrm{~h}$, monitored by TLC. It was then cooled by ice-water, filtered, and washed with water and the separated solid was then crystalized from benzene-petroleum ether as crystals. 


\subsubsection{2,3-Dithiophenylquinoxaline (2a)}

Yellow crystals, $1.47 \mathrm{~g}(85 \%)$ yield; m.p. $112-115^{\circ} \mathrm{C} . \mathrm{IR}(\mathrm{KBr}), 3052\left(\mathrm{sp}^{2}=\mathrm{C}-\mathrm{H}\right), 1580(\mathrm{C}=\mathrm{N})$ and 749, 682 (C-S, asymmetric and symmetric stretching) $\mathrm{cm}^{-1} .{ }^{1} \mathrm{H}-\mathrm{NMR}\left(500 \mathrm{MHz}, \mathrm{CDCl}_{3}, 300 \mathrm{~K}\right)$, $\delta$ 7.67-7.62 (m, 8H, Ar-H) and 7.46-7.48 (m, 6H, Ar-H) ppm. ${ }^{13} \mathrm{C}-\mathrm{NMR}$ (APT) (125 MHz, CDCl $\mathrm{M}_{3}$ $300 \mathrm{~K}), \delta 153.73(\mathrm{C}), 140.37(\mathrm{C}), 135.08(\mathrm{CH}), 129.09(\mathrm{CH}), 128.70(\mathrm{C}), 128.32(\mathrm{CH}), 127.88(\mathrm{CH}) \mathrm{ppm}$. $\mathrm{C}_{20} \mathrm{H}_{14} \mathrm{~N}_{2} \mathrm{~S}_{2}$ requires: $\mathrm{C}, 69.36 \% ; \mathrm{H}, 4.05 \%$;, $8.09 \%$. Found: $\mathrm{C}, 69.14 \% ; \mathrm{H}, 4.27 \% ; \mathrm{N}, 8.31 \%$.

\subsubsection{2,3-Di(thio-4-fluorophenyl)quinoxaline (2b)}

Colourless crystals, $2.13 \mathrm{~g}(93 \%)$ yield; m.p. 147-150 ${ }^{\circ} \mathrm{C}$. IR (KBr), $3037(=\mathrm{C}-\mathrm{H}), 1586(\mathrm{C}=\mathrm{N})$ and 752,636 (C-S, asymmetric and symmetric stretching) $\mathrm{cm}^{-1} .{ }^{1} \mathrm{H}-\mathrm{NMR}\left(500 \mathrm{MHz}, \mathrm{CDCl}_{3}, 300 \mathrm{~K}\right), \delta 7.62$ $(\mathrm{m}, 6 \mathrm{H}, \mathrm{Ar}-\mathrm{H}), 7.47\left(\mathrm{dd}, 2 \mathrm{H}, J=6.3,3.4 \mathrm{~Hz}, \mathrm{H}_{8}\right)$ and $7.16(\mathrm{t}, 4 \mathrm{H}, J=8.6 \mathrm{~Hz}, \mathrm{Ar}-\mathrm{H}) \mathrm{ppm} .{ }^{13} \mathrm{C}-\mathrm{NMR}$ (APT) $\left(125 \mathrm{MHz}, \mathrm{CDCl}_{3}, 300 \mathrm{~K}\right), \delta 163.53\left(\mathrm{~d},{ }^{1} J_{\mathrm{C}, \mathrm{F}}=250.3 \mathrm{~Hz}, \mathrm{CF}\right), 153.41(\mathrm{C}), 140.35$ (C), 137.49 $\left(\mathrm{d},{ }^{3} J_{\mathrm{C}, \mathrm{F}}=8.5 \mathrm{~Hz}, \mathrm{CH}\right), 128.60(\mathrm{CH}), 127.87(\mathrm{CH}), 123.52\left(\mathrm{~d},{ }^{4} J_{\mathrm{C}, \mathrm{F}}=2.5 \mathrm{~Hz}, \mathrm{C}\right), 116.47\left(\mathrm{~d},{ }^{2} J_{\mathrm{C}, \mathrm{F}}=22.2 \mathrm{~Hz}\right.$, $\mathrm{CH}$ ) ppm. $\mathrm{C}_{20} \mathrm{H}_{12} \mathrm{~N}_{2} \mathrm{~S}_{2} \mathrm{~F}_{2}$ requires: C, 52.63\%; H, 2.63\%; N, 6.14\%. Found: C, 55.47\%; H, 2.87\%; $\mathrm{N}, 6.27 \%$.

\subsubsection{2,3-Di(thio-4-bromophenyl)quinoxaline (2c)}

Colorless crystals, $2.3 \mathrm{~g}(94 \%)$ yield; m.p. $212-215^{\circ} \mathrm{C}$. IR $(\mathrm{KBr}), 3058\left(\mathrm{sp}^{2}=\mathrm{C}-\mathrm{H}\right), 1629(\mathrm{C}=\mathrm{N})$, and 758, 622 (C-S, asymmetric and symmetric stretching) $\mathrm{cm}^{-1} .{ }^{1} \mathrm{H}-\mathrm{NMR}\left(500 \mathrm{MHz}, \mathrm{CDCl}_{3}, 300 \mathrm{~K}\right)$, $\delta 7.67\left(\mathrm{dd}, 2 \mathrm{H}, J=6.3,3.5 \mathrm{~Hz}, \mathrm{H}_{8}\right), 7.58\left(\mathrm{~d}, 4 \mathrm{H}, J=8.4 \mathrm{~Hz}, \mathrm{H}_{3}{ }^{\prime}\right.$ and $\left.\mathrm{H}_{5}{ }^{\prime}\right)$, and $7.50\left(\mathrm{~m}, 6 \mathrm{H}_{1} \mathrm{H}_{7}, \mathrm{H}_{2}{ }^{\prime}\right.$, and $\left.\mathrm{H}_{6}{ }^{\prime}\right)$ ppm. ${ }^{13} \mathrm{C}-\mathrm{NMR}(\mathrm{APT})\left(125 \mathrm{MHz}: \mathrm{CDCl}_{3}\right), \delta 152.99(\mathrm{C}), 140.40(\mathrm{C}), 136.59(\mathrm{CH}), 132.39(\mathrm{CH})$, $128.84(\mathrm{CH}), 127.92(\mathrm{CH}), 127.66(\mathrm{C}), 123.87(\mathrm{C})$ ppm. $\mathrm{C}_{20} \mathrm{H}_{12} \mathrm{~N}_{2} \mathrm{~S}_{2} \mathrm{Br}_{2}$ requires: $\mathrm{C}, 47.62 \% ; \mathrm{H}, 2.38 \%$; N, 5.56\%. Found: C, $47.89 \% ; H, 2.54 \% ;$ N, 5.73\%.

\subsubsection{2,3-Di(thio-4-chlorophenyl)quinoxaline (2d)}

Pale yellow crystals, $1.87 \mathrm{~g}(90 \%)$ yield; m.p. $198-200^{\circ} \mathrm{C}$. IR $(\mathrm{KBr}), 3103\left(\mathrm{sp}^{2}=\mathrm{C}-\mathrm{H}\right), 1631(\mathrm{C}=\mathrm{N})$ and 756, 625 (C-S, asymmetric and symmetric stretching) $\mathrm{cm}^{-1} .{ }^{1} \mathrm{H}-\mathrm{NMR}\left(500 \mathrm{MHz}, \mathrm{CDCl}_{3}, 300 \mathrm{~K}\right)$, $\delta 7.66\left(\mathrm{dd}, 2 \mathrm{H}, \mathrm{J}=3.5 \mathrm{~Hz}, \mathrm{H}_{8}\right), 7.56\left(\mathrm{~d}, 4 \mathrm{H}, J=8.5 \mathrm{~Hz}, \mathrm{H}_{3}{ }^{\prime}\right.$ and $\left.\mathrm{H}_{5}{ }^{\prime}\right), 7.49\left(\mathrm{dd}, 2 \mathrm{H}, J=3.4 \mathrm{~Hz}, \mathrm{H}_{7}\right)$, and 7.43 $\left(\mathrm{d}, 4 \mathrm{H}, J=8.5 \mathrm{~Hz}, \mathrm{H}_{2}{ }^{\prime}\right.$, and $\left.\mathrm{H}_{6}{ }^{\prime}\right)$ ppm. ${ }^{13} \mathrm{C}-\mathrm{NMR}$ (APT) (125 MHz, $\left.\mathrm{CDCl}_{3}, 300 \mathrm{~K}\right), \delta 153.05(\mathrm{C}), 140.39$ (C), $136.41(\mathrm{CH}), 135.57(\mathrm{C}), 129.36(\mathrm{CH}), 128.78(\mathrm{CH}), 127.90(\mathrm{CH}), 126.97(\mathrm{C}) \mathrm{ppm} . \mathrm{C}_{20} \mathrm{H}_{12} \mathrm{~N}_{2} \mathrm{~S}_{2} \mathrm{Cl}_{2}$ requires: C, 57.83\%; H, 2.89\%; N, 6.75\%. Found: C, 57.61\%; H, 3.11\%; N, 6.89\%.

\subsubsection{2,3-Di(thio-4-methylphenyl)quinoxaline (2e)}

Colorless crystals, $1.59 \mathrm{~g}(85 \%)$ yield; m.p. $150{ }^{\circ} \mathrm{C} . \mathrm{IR}(\mathrm{KBr}), 3049$ (sp $\left.{ }^{2}=\mathrm{C}-\mathrm{H}\right), 2968\left(\mathrm{sp}^{3}-\mathrm{C}-\mathrm{H}\right)$, $1596(\mathrm{C}=\mathrm{N})$, and 751, $592\left(\mathrm{C}-\mathrm{S}\right.$, asymmetric and symmetric stretching) $\mathrm{cm}^{-1}$. ${ }^{1} \mathrm{H}-\mathrm{NMR}(500 \mathrm{MHz}$, $\left.\mathrm{CDCl}_{3}, 300 \mathrm{~K}\right), 7.65\left(\mathrm{dd}, 4 \mathrm{H}, J=6.3,3.5 \mathrm{~Hz}, \mathrm{H}_{8}\right), 7.53\left(\mathrm{~d}, 4 \mathrm{H}, J=8.1 \mathrm{~Hz}, \mathrm{H}_{2}{ }^{\prime}\right.$, and $\left.\mathrm{H}_{6}{ }^{\prime}\right), 7.44(\mathrm{dd}, 2 \mathrm{H}$, $\left.J=6.4,3.4 \mathrm{~Hz}, \mathrm{H}_{7}\right), 7.27\left(\mathrm{~d}, 4 \mathrm{H}, J=8.0 \mathrm{~Hz}, \mathrm{H}_{3}{ }^{\prime}\right.$ and $\left.\mathrm{H}_{5}{ }^{\prime}\right)$, and $2.42\left(\mathrm{~s}, 6 \mathrm{H}, 2 \mathrm{CH}_{3}\right)$ ppm. ${ }^{13} \mathrm{C}-\mathrm{NMR}(\mathrm{APT})$ (125 MHz, $\left.\mathrm{CDCl}_{3}, 300 \mathrm{~K}\right), \delta 154.01$ (C), 140.32 (C), $139.26(\mathrm{C}), 135.13(\mathrm{CH}), 129.96(\mathrm{CH}), 128.19(\mathrm{CH})$, $127.83(\mathrm{CH}), 124.95(\mathrm{C}), 21.28\left(\mathrm{CH}_{3}\right)$ ppm. $\mathrm{C}_{22} \mathrm{H}_{18} \mathrm{~N}_{2} \mathrm{~S}_{2}$ requires: $\mathrm{C}, 70.5 \% ; \mathrm{H}, 4.8 \%$; N, 7.4\%. Found: C, $70.78 \% ; \mathrm{H}, 4.73 \% ; \mathrm{N}, 7.68 \%$.

\subsection{Synthesis of $N^{2}, N^{3}$-Diarylquinoxaline-2,3-diamine (3a-g)}

In a typical experiment a solution of 2,3-DCQ $1(0.5 \mathrm{~g}, 0.0025 \mathrm{~mol})$ and $0.025 \mathrm{~mol}$ of appropriate amine; (namely, 2-aminopyridine (2.35 g), aniline (2.33 g), $m$-chloroaniline (3.19 g), p-toludine (2.68 g), 3,4-dimethylaniline (3.03 g), p-anisidine (3.08 g) or 3,4-dimethoxyaniline (3.83 g)) and triethylamine $(0.5 \mathrm{~g}, 0.005 \mathrm{~mol})$ in $20 \mathrm{~mL}$ acetonitrile was refluxed for 20 to $40 \mathrm{~h}$ with constant stirring, monitored by TLC. The reaction mixture was then cooled by ice-water, filtered, washed with water to give quinoxalines $\mathbf{3 a -} \mathbf{g}$ as solid crystals which were purified by crystallization from alcohol. 
(Note: as an exception the reaction of 2-aminopyridine with 2,3-DCQ was carried out in dimethylformamide instead of acetonitrile).

\subsection{1. $\mathrm{N}^{2}, \mathrm{~N}^{3}$-Di(pyridin-2-yl)quinoxaline-2,3-diamine (3a)}

Yellow crystals, $0.59 \mathrm{~g}(75 \%)$ yield; m.p. $>250{ }^{\circ} \mathrm{C}$. IR (KBr), $3372(\mathrm{NH}), 3107$ (=C-H), and 1667 $(\mathrm{C}=\mathrm{N}) \mathrm{cm}^{-1} .{ }^{1} \mathrm{H}-\mathrm{NMR}\left(500 \mathrm{MHz}, \mathrm{DMSO}-d_{6}, 300 \mathrm{~K}\right), \delta 11.96$ (broad s, $1 \mathrm{H}, \mathrm{NH}, \mathrm{D}_{2} \mathrm{O}$ exchangeable), 11.91 (broad s, 2H, NH, $\mathrm{D}_{2} \mathrm{O}$ exchangeable), 7.38-7.26 (m, 2H, Ar-H), and 7.15-7.02 (m, 10H, Ar-H) ppm. 13C-NMR (APT) (125 MHz, DMSO- $\left.d_{6}, 300 \mathrm{~K}\right), \delta 132.82$ (C), $129.03(\mathrm{C}), 125.60(\mathrm{C}), 124.63(\mathrm{CH})$, $123.78(\mathrm{CH}), 123.04(\mathrm{CH}), 123.01(\mathrm{CH}), 115.13(\mathrm{CH}), 114.22(\mathrm{CH})$ ppm.C18H14N6 requires: $\mathrm{C}, 68.7 \%$; $\mathrm{H}$, $4.4 \%$; N $26.7 \%$. Found: C, $68.99 \%$; H, $4.63 \%$; N $26.43 \%$.

\subsection{2. $\mathrm{N}^{2}, \mathrm{~N}^{3}$-Diphenylquinoxaline-2,3-diamine (3b)}

Yellow crystals, $1.31 \mathrm{~g}(84 \%)$ yield; m.p. $228-230{ }^{\circ} \mathrm{C}$ (Lit. $\left.223{ }^{\circ} \mathrm{C}\right)$ [45]. IR (KBr), $3311(\mathrm{NH})$, $3075(=\mathrm{C}-\mathrm{H})$, and $1642(\mathrm{C}=\mathrm{N}) \mathrm{cm}^{-1} .{ }^{1} \mathrm{H}-\mathrm{NMR}\left(500 \mathrm{MHz}\right.$, DMSO-d $\left.d_{6}, 300 \mathrm{~K}\right), \delta 9.63$ (br. s, 2H, NH, $\mathrm{D}_{2} \mathrm{O}$ exchangeable), $7.93\left(\mathrm{~d}, 4 \mathrm{H}, J=7.9 \mathrm{~Hz}, \mathrm{H}_{2}{ }^{\prime}\right.$, and $\left.\mathrm{H}_{6}{ }^{\prime}\right), 7.55\left(\mathrm{dd}, 2 \mathrm{H}, J=6.2,3.4 \mathrm{~Hz}, \mathrm{H}_{8}\right), 7.41\left(\mathrm{t}, 4 \mathrm{H}, \mathrm{H}_{3}{ }^{\prime}\right.$ and $\left.\mathrm{H}_{5}{ }^{\prime}\right), 7.34\left(\mathrm{dd}, 2 \mathrm{H}, J=6.2,3.4 \mathrm{~Hz}, \mathrm{H}_{7}\right)$, and $7.10\left(\mathrm{t}, 2 \mathrm{H}, J=7.4 \mathrm{~Hz}, \mathrm{H}_{4}{ }^{\prime}\right)$ ppm. ${ }^{13} \mathrm{C}-\mathrm{NMR}$ (APT) $\left(125 \mathrm{MHz}, \mathrm{DMSO}-d_{6}, 300 \mathrm{~K}\right), \delta 141.32(\mathrm{C}), 139.37(\mathrm{C}), 133.94(\mathrm{C}), 128.93(\mathrm{CH}), 125.66(\mathrm{CH}), 124.13(\mathrm{CH})$, $123.79(\mathrm{CH}), 121.53(\mathrm{CH})$ ppm. $\mathrm{C}_{20} \mathrm{H}_{16} \mathrm{~N}_{4}$ requires: $\mathrm{C}, 76.92 \% ; \mathrm{H}, 5.13 \% ; \mathrm{N}, 17.95 \%$. Found: $\mathrm{C}, 77.12 \%$; $\mathrm{H}, 5.29 \%$;, $18.24 \%$.

\subsection{3. $\mathrm{N}^{2}, \mathrm{~N}^{3}$-Bis(3-chlorophenyl)quinoxaline-2,3-diamine (3c)}

Yellow crystals, $0.75 \mathrm{~g}(81 \%)$ yield; m.p. 122-124 ${ }^{\circ} \mathrm{C}$. IR (KBr), $3303(\mathrm{NH}), 3054(=\mathrm{C}-\mathrm{H})$, and 1575 $(\mathrm{C}=\mathrm{N}) \mathrm{cm}^{-1} .{ }^{1} \mathrm{H}-\mathrm{NMR}\left(500 \mathrm{MHz}, \mathrm{DMSO}-d_{6}, 300 \mathrm{~K}\right), \delta 9.16\left(\mathrm{~s}, 2 \mathrm{H}, \mathrm{NH}, \mathrm{D}_{2} \mathrm{O}\right.$ exchangeable), $8.09(\mathrm{t}, 2 \mathrm{H}$, $\left.J=2.0 \mathrm{~Hz}, \mathrm{H}_{5}{ }^{\prime}\right), 7.81\left(\mathrm{dd}, 2 \mathrm{H}, J=8.2,1.5 \mathrm{~Hz}, \mathrm{H}_{4}{ }^{\prime}\right), 7.59\left(\mathrm{dd}, 2 \mathrm{H}, J=6.1,3.4 \mathrm{~Hz}, \mathrm{H}_{8}\right), 7.44-7.38\left(\mathrm{~m}, 4 \mathrm{H}, \mathrm{H}_{7}\right.$, and $\left.\mathrm{H}_{2}{ }^{\prime}\right)$, and $7.11\left(\mathrm{dd}, 2 \mathrm{H}, J=7.9,1.6 \mathrm{~Hz}, \mathrm{H}_{6}{ }^{\prime}\right)$ ppm. ${ }^{13} \mathrm{C}-\mathrm{NMR}$ (APT) $\left(125 \mathrm{MHz}\right.$, DMSO- $\left.d_{6}, 300 \mathrm{~K}\right), \delta$ 141.76 (C), $140.95(\mathrm{C}), 136.04(\mathrm{C}), 132.95(\mathrm{C}), 130.27(\mathrm{CH}), 125.81(\mathrm{CH}), 125.60(\mathrm{CH}), 122.03(\mathrm{CH}), 119.50$ $(\mathrm{CH}), 118.52(\mathrm{CH})$ ppm. $\mathrm{C}_{20} \mathrm{H}_{14} \mathrm{~N}_{4} \mathrm{~S}_{2} \mathrm{Cl}_{2}$ requires: $\mathrm{C}, 62.99 \%$; $\mathrm{H}, 3.67 \%$; N, $14.70 \%$. Found: $\mathrm{C}, 62.84 \%$; $\mathrm{H}$, $3.75 \% ; \mathrm{N}, 14.81 \%$.

\subsection{4. $\mathrm{N}^{2}, \mathrm{~N}^{3}$-Dip-tolylquinoxaline-2,3-diamine (3d)}

Yellow crystals, $0.67 \mathrm{~g}(79 \%)$ yield; m.p. $145-147^{\circ} \mathrm{C}$ (Lit. 147-149 $\left.{ }^{\circ} \mathrm{C}\right)$ [46]. IR (KBr), $3388(\mathrm{NH})$, $3026(=\mathrm{C}-\mathrm{H}), 2916(-\mathrm{C}-\mathrm{H})$, and $1640(\mathrm{C}=\mathrm{N}) \mathrm{cm}^{-1} .{ }^{1} \mathrm{H}-\mathrm{NMR}\left(500 \mathrm{MHz}, \mathrm{DMSO}-d_{6}, 300 \mathrm{~K}\right), \delta 8.92$ (br. s, $2 \mathrm{H}, \mathrm{NH}, \mathrm{D}_{2} \mathrm{O}$ exchangeable), $7.76\left(\mathrm{~d}, 4 \mathrm{H}, J=8.4 \mathrm{~Hz}, \mathrm{H}_{2}{ }^{\prime}\right.$, and $\left.\mathrm{H}_{6}{ }^{\prime}\right), 7.49\left(\mathrm{dd}, 2 \mathrm{H}, J=6.1,3.5 \mathrm{~Hz}, \mathrm{H}_{8}\right)$, $7.30\left(\mathrm{dd}, 2 \mathrm{H}, J=6.0,3.4 \mathrm{~Hz}, \mathrm{H}_{7}\right), 7.20\left(\mathrm{~d}, 4 \mathrm{H}, J=8.3 \mathrm{~Hz}, \mathrm{H}_{3}{ }^{\prime}\right.$, and $\left.\mathrm{H}_{5}{ }^{\prime}\right)$, and $2.30\left(\mathrm{~s}, 6 \mathrm{H}, 2 \mathrm{CH}_{3}\right) \mathrm{ppm}$. ${ }^{13} \mathrm{C}-\mathrm{NMR}$ (APT) $\left(125 \mathrm{MHz}, \mathrm{DMSO}-d_{6}, 300 \mathrm{~K}\right), \delta 141.21$ (C), 137.57 (C), 136.16 (C), 131.58 (C), 129.05 $(\mathrm{CH}), 125.25(\mathrm{CH}), 124.89(\mathrm{CH}), 120.77(\mathrm{CH}), 20.51\left(\mathrm{CH}_{3}\right)$ ppm. $\mathrm{C}_{22} \mathrm{H}_{20} \mathrm{~N}_{4}$ requires: $\mathrm{C}, 77.65 \%$; $\mathrm{H}, 5.88 \%$; N, 16.47\%. Found: C, 77.47\%; H, 5.98\%; N, 16.62\%.

\subsection{5. $\mathrm{N}^{2}, \mathrm{~N}^{3}$-Bis(3,4-dimethylphenyl)quinoxaline-2,3-diamine (3e)}

Yellow crystals, $0.38 \mathrm{~g}(84 \%)$ yield; m.p. $258-261{ }^{\circ} \mathrm{C}$. IR (KBr), $3390(\mathrm{NH}), 3154(=\mathrm{C}-\mathrm{H}), 2881$ $\left(\mathrm{sp}^{3}-\mathrm{C}-\mathrm{H}\right)$, and $1634(\mathrm{C}=\mathrm{N}) \mathrm{cm}^{-1} .{ }^{1} \mathrm{H}-\mathrm{NMR}\left(500 \mathrm{MHz}, \mathrm{DMSO}-d_{6}, 300 \mathrm{~K}\right): \delta 9.85\left(\mathrm{~s}, 2 \mathrm{H}, \mathrm{NH}, \mathrm{D}_{2} \mathrm{O}\right.$ exchangeable), 7.70-7.58 (m, 4H, Ar-H), $7.55\left(\mathrm{dd}, 2 \mathrm{H}, J=6.0,3.5 \mathrm{~Hz}, \mathrm{H}_{8}\right), 7.32(\mathrm{dd}, 2 \mathrm{H}, J=6.0,3.4 \mathrm{~Hz}$, $\left.\mathrm{H}_{7}\right), 7.19(\mathrm{~d}, 2 \mathrm{H}, \mathrm{Ar}-\mathrm{H}), 2.26\left(\mathrm{~s}, 6 \mathrm{H}, 2 \mathrm{CH}_{3}\right)$, and $2.23\left(\mathrm{~s}, 6 \mathrm{H}, 2 \mathrm{CH}_{3}\right) \mathrm{ppm} .{ }^{13} \mathrm{C}-\mathrm{NMR}$ (APT) $(125 \mathrm{MHz}$, DMSO- $\left.d_{6}, 300 \mathrm{~K}\right): \delta 141.18(\mathrm{C}), 136.54(\mathrm{C}), 131.81(\mathrm{C}), 129.81(\mathrm{CH}), 127.80(\mathrm{C}), 126.17(\mathrm{C}), 125.34(\mathrm{CH})$, $122.83(\mathrm{CH}), 119.21(\mathrm{CH}), 118,72(\mathrm{CH}), 19.72\left(\mathrm{CH}_{3}\right), 18.89\left(\mathrm{CH}_{3}\right)$ ppm. $\mathrm{C}_{24} \mathrm{H}_{24} \mathrm{~N}_{4}$ requires: $\mathrm{C}, 91.3 \% ; \mathrm{H}$, $6.52 \% ; \mathrm{N}, 15.2 \%$. Found: $\mathrm{C}, 78.45 \%$; $\mathrm{H}, 6.71 \%$;, $15.47 \%$. 
3.7.6. $\mathrm{N}^{2}, \mathrm{~N}^{3}$-bis(4-methoxyphenyl)quinoxaline-2,3-diamine (3f)

Green crystals, $0.72 \mathrm{~g}(78 \%)$ yield; m.p. 116-118 ${ }^{\circ} \mathrm{C}$. IR (KBr): $3387(\mathrm{NH}), 3070$ (=C-H), 2949 (-C-H), and $1656(\mathrm{C}=\mathrm{N}) \mathrm{cm}^{-1} .{ }^{1} \mathrm{H}-\mathrm{NMR}\left(500 \mathrm{MHz}, \mathrm{DMSO}-d_{6}, 300 \mathrm{~K}\right): \delta 8.84$ (br. s, $2 \mathrm{H}, \mathrm{NH}, \mathrm{D}_{2} \mathrm{O}$ exchangeable), $7.77\left(\mathrm{~d}, 4 \mathrm{H}, J=9.0 \mathrm{~Hz}, \mathrm{H}_{2}{ }^{\prime}\right.$, and $\left.\mathrm{H}_{6}{ }^{\prime}\right), 7.45\left(\mathrm{dd}, 2 \mathrm{H}, J=6.2,3.5 \mathrm{~Hz}, \mathrm{H}_{8}\right), 7.26(\mathrm{dd}, 2 \mathrm{H}, J=6.0,3.4 \mathrm{~Hz}$, $\left.\mathrm{H}_{7}\right), 6.98\left(\mathrm{~d}, 4 \mathrm{H}, J=9.0 \mathrm{~Hz}, \mathrm{H}_{3}{ }^{\prime}\right.$, and $\left.\mathrm{H}_{5}{ }^{\prime}\right)$, and $3.76\left(\mathrm{~s}, 6 \mathrm{H}, 2 \mathrm{OCH}_{3}\right) \mathrm{ppm} .{ }^{13} \mathrm{C}-\mathrm{NMR}$ (APT) $(125 \mathrm{MHz}$, DMSO- $\left.d_{6}, 300 \mathrm{~K}\right): \delta 155.06(\mathrm{C}), 141.33(\mathrm{C}), 136.21(\mathrm{C}), 133.11(\mathrm{C}), 125.12(\mathrm{CH}), 124.66(\mathrm{CH}), 122.48(\mathrm{CH})$, $113.87(\mathrm{CH}), 55.22\left(\mathrm{OCH}_{3}\right)$ ppm. $\mathrm{C}_{22} \mathrm{H}_{20} \mathrm{~N}_{4} \mathrm{O}_{2}$ requires: $\mathrm{C}, 70.97 \% ; \mathrm{H}, 5.38 \% ; \mathrm{N}, 15.05 \%$. Found: $\mathrm{C}$, $70.73 \% ; \mathrm{H}, 5.47 \% ; \mathrm{N}, 14.89 \%$.

\subsection{7. $\mathrm{N}^{2}, \mathrm{~N}^{3}$-Bis(3,4-dimethoxyphenyl)quinoxaline-2,3-diamine (3g)}

Dark green crystals, $0.41 \mathrm{~g}(76 \%)$ yield; m.p. $208-210{ }^{\circ} \mathrm{C}$ (Lit. $\left.214{ }^{\circ} \mathrm{C}\right) . \mathrm{IR}(\mathrm{KBr}): 3372(\mathrm{NH}), 3059$ $(=\mathrm{C}-\mathrm{H}), 2936(-\mathrm{C}-\mathrm{H})$, and $1615(\mathrm{C}=\mathrm{N}) \mathrm{cm}^{-1} .{ }^{1} \mathrm{H}-\mathrm{NMR}\left(500 \mathrm{MHz}, \mathrm{DMSO}-d_{6}, 300 \mathrm{~K}\right): \delta 8.87$ (br. s, 2H, $\mathrm{NH}, \mathrm{D}_{2} \mathrm{O}$ exchangeable), $7.66\left(\mathrm{~d}, 2 \mathrm{H}, J=2.4 \mathrm{~Hz}, \mathrm{H}_{2}{ }^{\prime}\right), 7.49\left(\mathrm{dd}, 2 \mathrm{H}, J=6.2,3.5 \mathrm{~Hz}, \mathrm{H}_{8}\right), 7.38(\mathrm{dd}, 2 \mathrm{H}$, $\left.J=8.6,2.4 \mathrm{~Hz}_{1} \mathrm{H}_{6}{ }^{\prime}\right), 7.29\left(\mathrm{dd}, 2 \mathrm{H}, J=6.1,3.4 \mathrm{~Hz}, \mathrm{H}_{7}\right), 6.99\left(\mathrm{~d}, 2 \mathrm{H}, J=8.7 \mathrm{~Hz}, \mathrm{H}_{5}{ }^{\prime}\right), 3.81\left(\mathrm{~s}, 6 \mathrm{H}, 2 \mathrm{OCH}_{3}\right)$, and $3.76\left(\mathrm{~s}, 6 \mathrm{H}, 2 \mathrm{OCH}_{3}\right)$ ppm. ${ }^{13} \mathrm{C}-\mathrm{NMR}(\mathrm{APT})\left(125 \mathrm{MHz}, \mathrm{DMSO}-d_{6}, 300 \mathrm{~K}\right): \delta 148.49(\mathrm{C}), 144.56(\mathrm{C})$, $141.18(\mathrm{C}), 136.09(\mathrm{C}), 133.63(\mathrm{C}), 125.21(\mathrm{CH}), 124.79(\mathrm{CH}), 112.61(\mathrm{CH}), 112.06(\mathrm{CH}), 106.05(\mathrm{CH}), 55.78$ $\left(\mathrm{OCH}_{3}\right), 55.40\left(\mathrm{OCH}_{3}\right)$ ppm. $\mathrm{C}_{24} \mathrm{H}_{24} \mathrm{~N}_{4} \mathrm{O}_{4}$ requires: $\mathrm{C}, 66.6 \% ; \mathrm{H}, 5.55 \% ; \mathrm{N}, 12.9 \%$. Found: $\mathrm{C}, 66.87 \% ; \mathrm{H}$, $5.62 \% ; \mathrm{N}, 12.78 \%$. Spectral data of $3 \mathrm{~g}$ are in accordance with those reported in the literature [47].

\subsection{Synthesis and Characterization of 2,2'-(Quinoxaline-2,3-diylbis(sulfanediyl))diacetic acid (4)}

A solution of 2,3-DCQ 1 (1 g, $0.005 \mathrm{~mol})$ and a solution of thioglycolic acid $(1.38 \mathrm{~g}, 0.015 \mathrm{~mol})$ and triethyl amine $(3.03 \mathrm{~g}, 0.03 \mathrm{~mol})$ in $30 \mathrm{~mL}$ methanol was refluxed for $4 \mathrm{~h}$. The reaction was monitored by TLC until 2,3-DCQ was totally consumed. The reaction mixture was then cooled by ice-water and the separated solid was filtered then crystalized from a mixture of methanol and water.

Yellow crystals, $1.78 \mathrm{~g}(93 \%)$ yield; m.p. 227-229 ${ }^{\circ} \mathrm{C}$. IR (KBr): $3426(\mathrm{OH}), 2999$ (-C-H), 1705 (C=O), $1560(\mathrm{C}=\mathrm{N})$, and 759, 675 (C-S, asymmetric and symmetric stretching) $\mathrm{cm}^{-1} .{ }^{1} \mathrm{H}-\mathrm{NMR}(500 \mathrm{MHz}$, DMSO- $\left.d_{6}, 300 \mathrm{~K}\right): \delta 7.83\left(\mathrm{dd}, 2 \mathrm{H}, J=6.3,3.4 \mathrm{~Hz}, \mathrm{H}_{8}\right), 7.67\left(\mathrm{dd}, 2 \mathrm{H}, J=6.2,3.4 \mathrm{~Hz}, \mathrm{H}_{7}\right)$, and $4.17(\mathrm{~s}, 4 \mathrm{H}$, $\left.2 \mathrm{CH}_{2}\right)$ ppm. ${ }^{13} \mathrm{C}-\mathrm{NMR}(\mathrm{APT})\left(125 \mathrm{MHz}, \mathrm{DMSO}-d_{6}, 300 \mathrm{~K}\right): \delta 169.53(\mathrm{CO}), 152.60$ (C), 138.97 (C), 128.94 $(\mathrm{CH}), 127.16(\mathrm{CH}), 32.49\left(\mathrm{CH}_{2}\right)$ ppm. $\mathrm{C}_{12} \mathrm{H}_{10} \mathrm{~N}_{2} \mathrm{~S}_{2} \mathrm{O}_{4}$ requires: $\mathrm{C}, 37.6 \% ; \mathrm{H}, 2.6 \% ; \mathrm{N}, 7.3 \%$. Found: $\mathrm{C}$, $37.75 \% ; \mathrm{H}, 2.47 \% ; \mathrm{N}: 7.49 \%$.

\subsection{Synthesis and Characterization of 3-Chloro-N-(4-chlorophenyl)quinoxalin-2-amine (5)}

To a solution of $p$-chloroaniline $(3.18 \mathrm{~g}, 0.025 \mathrm{~mol})$ and triethylamine $(0.5 \mathrm{~g}, 0.005 \mathrm{~mol})$ in $5 \mathrm{~mL}$ dimethylformamide, a solution of 2,3-DCQ $1(0.5 \mathrm{~g}, 0.0025 \mathrm{~mol})$ in $10 \mathrm{~mL}$ dimethylformamide was added with constant stirring. After the completion of addition, the reaction mixture was refluxed for $40 \mathrm{~h}$, cooled by ice-water, filtered, washed with water, and the product formed was purified by crystallization from hot ethanol. Yellow crystals, $0.55 \mathrm{~g}$ (76\%) yield; m.p. $227-229^{\circ} \mathrm{C}$. IR (KBr): 3357 $(\mathrm{NH}), 3099(=\mathrm{C}-\mathrm{H})$, and $1676(\mathrm{C}=\mathrm{N}) \mathrm{cm}^{-1} .{ }^{1} \mathrm{H}-\mathrm{NMR}\left(500 \mathrm{MHz}, \mathrm{DMSO}-d_{6}, 300 \mathrm{~K}\right): \delta 9.13($ br. s, $1 \mathrm{H}$, $\mathrm{NH}, \mathrm{D}_{2} \mathrm{O}$ exchangeable), $7.92\left(\mathrm{~d}, 2 \mathrm{H}, J=8.9 \mathrm{~Hz}, \mathrm{H}_{2}{ }^{\prime}\right.$ and $\left.\mathrm{H}_{6}{ }^{\prime}\right), 7.56(\mathrm{dd}, 1 \mathrm{H}, J=6.0,3.5 \mathrm{~Hz}, \mathrm{Ar}-\mathrm{H}), 7.44$ $\left(\mathrm{d}, 2 \mathrm{H}, J=8.8 \mathrm{~Hz}, \mathrm{H}_{3}{ }^{\prime}\right.$, and $\left.\mathrm{H}_{5}{ }^{\prime}\right), 7.41-7.30(\mathrm{~m}, 2 \mathrm{H}, \mathrm{Ar}-\mathrm{H})$, and 7.28-7.12 (m, 1H, Ar-H) ppm. ${ }^{13} \mathrm{C}-\mathrm{NMR}$ (APT) $\left(125 \mathrm{MHz}, \mathrm{DMSO}-d_{6}, 300 \mathrm{~K}\right): \delta 151.43(\mathrm{C}), 140.57$ (C), 138.97 (C), 138.63 (C), 135.96 (C), 128.51 $(\mathrm{CH}), 128.36(\mathrm{CH}), 125.89(\mathrm{C}), 125.52(\mathrm{CH}), 121.91(\mathrm{CH}), 121.29(\mathrm{CH}), 115.08(\mathrm{CH}) \mathrm{ppm} . \mathrm{C}_{14} \mathrm{H}_{9} \mathrm{~N}_{3} \mathrm{Cl}_{2}$ requires: $\mathrm{C}, 57.9 \%$; $\mathrm{H}, 3.10 \%$; N $14.4 \%$. Found: $\mathrm{C}, 58.13 \%$; $\mathrm{H}, 3.29 \%$;, $14.67 \%$.

\subsection{Synthesis of 2-Chloro-3-(arylthio)quinoxaline (6a-b)}

A solution of 2,3-DCQ $1(1 \mathrm{~g}, 0.005 \mathrm{~mol})$ and appropriate thiol (Namely, thiophenol or thiosalicylic acid) $(0.015 \mathrm{~mol})$ and triethylamine $(1.01 \mathrm{~g}, 0.01 \mathrm{~mol})$ in $30 \mathrm{~mL}$ methanol was stirred for $4 \mathrm{~h}$, monitored by TLC. The reaction mixture was then cooled by ice-water, filtered, washed with water, and purified by crystallization. 


\subsubsection{2-Chloro-3-(phenylthio)quinoxaline (6a)}

Colorless crystals (benzene-petroleum ether), $1.25 \mathrm{~g}$ (92\%) yield; m.p. $145{ }^{\circ} \mathrm{C}$. IR (KBr): 3068 $(=\mathrm{C}-\mathrm{H}), 1562(\mathrm{C}=\mathrm{N})$, and 753, 683 (C-S, asymmetric and symmetric stretching) $\mathrm{cm}^{-1} .{ }^{1} \mathrm{H}-\mathrm{NMR}(500 \mathrm{MHz}$, $\left.\mathrm{CDCl}_{3}, 300 \mathrm{~K}\right): \delta$ 7.93-7.90 (m, 1H, Ar-H), 7.69-7.65 (m, 1H, Ar-H), 7.65-7.58 (m, 4H, Ar-H), and 7.51-7.46 (m, 3H, Ar-H) ppm. ${ }^{13} \mathrm{C}-\mathrm{NMR}$ (APT) (125 MHz, CDCl, $\left.300 \mathrm{~K}\right): \delta 155.30$ (C), 144.32 (C), 141.21 (C), $139.68(\mathrm{C}), 135.56(\mathrm{CH}), 130.10(\mathrm{CH}), 129.62(\mathrm{CH}), 129.28(\mathrm{CH}), 129.15(\mathrm{CH}), 128.12(\mathrm{C}), 128.01(\mathrm{CH})$, $127.98(\mathrm{CH})$ ppm. ${ }_{14} \mathrm{H}_{9} \mathrm{~N}_{2} \mathrm{SCl}$ requires: $\mathrm{C}, 61.65 \% ; \mathrm{H}, 3.30 \%$;, $10.28 \%$. Found: $\mathrm{C}, 61.92 \% ; \mathrm{H}, 3.19 \%$; N, $10.48 \%$.

\subsubsection{2-(3-Chloroquinoxalin-2-ylthio)benzoic Acid (6b)}

Dark yellow crystals (methanol-water mixture), $1.4 \mathrm{~g}(92 \%)$ yield; m.p. $>250{ }^{\circ} \mathrm{C}$. IR (KBr): $3430(\mathrm{OH}), 3062(=\mathrm{C}-\mathrm{H}), 1681(\mathrm{C}=\mathrm{O}), 1586(\mathrm{C}=\mathrm{N})$, and 744, $646(\mathrm{C}-\mathrm{S}$, asymmetric and symmetric stretching) $\mathrm{cm}^{-1} .{ }^{1} \mathrm{H}-\mathrm{NMR}\left(500 \mathrm{MHz}, \mathrm{DMSO}-d_{6}, 300 \mathrm{~K}\right): \delta 8.01(\mathrm{~d}, 1 \mathrm{H}, J=6.4 \mathrm{~Hz}, \mathrm{Ar}-\mathrm{H}), 7.98-7.93$ $(\mathrm{m}, 1 \mathrm{H}, \mathrm{Ar}-\mathrm{H}), 7.77(\mathrm{dd}, 1 \mathrm{H}, J=6.3,3.5 \mathrm{~Hz}, \mathrm{Ar}-\mathrm{H}), 7.70(\mathrm{~m}, 1 \mathrm{H}, \mathrm{Ar}-\mathrm{H}), 7.61$ (d, 1H, J = 7.9 Hz, Ar-H), 7.53 $(\mathrm{m}, 2 \mathrm{H}, \mathrm{Ar}-\mathrm{H})$, and $7.33(\mathrm{t}, 1 \mathrm{H}, J=7.9 \mathrm{~Hz}, \mathrm{Ar}-\mathrm{H}) \mathrm{ppm} .{ }^{13} \mathrm{C}-\mathrm{NMR}$ (APT) $\left(125 \mathrm{MHz}, \mathrm{DMSO}-d_{6}, 300 \mathrm{~K}\right)$ : $\delta 167.54(\mathrm{CO}), 153.65(\mathrm{C}), 139.95(\mathrm{C}), 138.84(\mathrm{C}), 135.69(\mathrm{CH}), 135.19(\mathrm{C}), 133.73(\mathrm{CH}), 133.65(\mathrm{C}), 133.19$ (CH), $131.50(\mathrm{CH}), 130.55(\mathrm{CH}), 128.40(\mathrm{C}), 128.00(\mathrm{CH}), 125.90(\mathrm{CH}), 124.90(\mathrm{CH}) \mathrm{ppm} . \mathrm{C}_{15} \mathrm{H}_{9} \mathrm{~N}_{2} \mathrm{SO}_{2} \mathrm{Cl}$ requires: C, 56.8\%; H, 2.8\%; N, 8.8\%. Found C, 56.97\%; H, 3.13\%; N, 8.61\%.

\subsection{Synthesis of 2-Chloro-3-hydrazinylquinoxaline (7)}

A solution of 2,3-DCQ 1 ( $5 \mathrm{~g}, 0.025 \mathrm{~mol})$ and hydrazine hydrate $(2.7 \mathrm{~g}, 0.0055 \mathrm{~mol})$ in $15 \mathrm{~mL}$ ethanol was stirred for $24 \mathrm{~h}$ with constant stirring, the reaction being monitored by TLC. The reaction mixture was then filtered and washed with water and the product was crystalized from benzene-petroleum ether as dark orang crystals, $82 \%$ yield; m.p. $>250{ }^{\circ} \mathrm{C}\left[\right.$ lit. $\left.>300{ }^{\circ} \mathrm{C}\right]$ the spectral data agreed with the previously reported data [48].

\subsection{Synthesis of 2-Hydrazinyl-3-(phenylthio)quinoxaline (8)}

To a solution of thiophenol $(7.00 \mathrm{~g}, 0.069 \mathrm{~mol})$ and $0.138 \mathrm{~mL}$ of triethylamine in $20 \mathrm{~mL}$ methanol, a solution of 2-chloro-3-hydrazinylquinoxaline $7(4.5 \mathrm{~g}, 0.023 \mathrm{~mol})$ in $20 \mathrm{~mL}$ methanol was added. Then the reaction mixture was refluxed for $30 \mathrm{~min}$, monitored by TLC, cooled by ice-water, filtered, washed with water, and purified by crystallization from benzene. Orange crystals, $6.3 \mathrm{~g}(92 \%)$ yield; m.p. $210-212{ }^{\circ} \mathrm{C}$. IR (KBr): $3300(\mathrm{NH}), 3237\left(\mathrm{NH}_{2}\right), 3033(=\mathrm{C}-\mathrm{H}), 1627(\mathrm{C}=\mathrm{N})$, and 750, 683 (C-S, asymmetric and symmetric stretching) $\mathrm{cm}^{-1}$. ${ }^{1} \mathrm{H}-\mathrm{NMR}\left(500 \mathrm{MHz}, \mathrm{DMSO}-d_{6}, 300 \mathrm{~K}\right): \delta 7.62-7.54$ $(\mathrm{m}, 1 \mathrm{H}, \mathrm{Ar}-\mathrm{H}), 7.52-7.49$ (m, 1H, Ar-H), 7.50-7.47 (m, 1H, Ar-H), 7.47-7.40 (m, 2H, Ar-H), 7.11 (t, 1H, $J=8.3 \mathrm{~Hz}, \mathrm{Ar}-\mathrm{H}), 7.02(\mathrm{~d}, 1 \mathrm{H}, J=8.9 \mathrm{~Hz}, \mathrm{Ar}-\mathrm{H}), 6.92(\mathrm{~d}, 1 \mathrm{H}, J=9.0 \mathrm{~Hz}, \mathrm{Ar}-\mathrm{H}), 6.80(\mathrm{t}, 1 \mathrm{H}, J=7.6 \mathrm{~Hz}$, Ar-H), 5.61 (br.s, $2 \mathrm{H}, \mathrm{NH}_{2}, \mathrm{D}_{2} \mathrm{O}$ exchangeable), and 4.58 (br.s, $1 \mathrm{H}, \mathrm{NH}, \mathrm{D}_{2} \mathrm{O}$ exchangeable). $\mathrm{C}_{14} \mathrm{H}_{12} \mathrm{~N}_{4} \mathrm{~S}$ requires: C, $62.67 \% ; \mathrm{H}, 4.48 \%$; N $20.90 \%$. Found: C, $62.43 \% ; \mathrm{H}, 4.59 \% ; \mathrm{N}, 21.16 \%$.

\subsection{Synthesis of (2-(2-Arylidenehydrazinyl)-3-(phenylthio)quinoxaline (9a-f)}

In a typical experiment a solution of 2-hydrazinyl-3-(phenylthio)quinoxaline 8 (0.5 g, $0.0018 \mathrm{~mol}$ ) and appropriate aldehyde $(0.0018 \mathrm{~mol})$ (Namely, benzaldehyde, p-nitrobenzaldehyde, m-nitrobenzaldehyde, p-methoxybenzaldehyde, p-chlorobenzaldehyde or p-hydroxybenzaldehyde) in ethanol-DMF (20-2 mL) was refluxed for $6 \mathrm{~h}$ with constant stirring, the reaction being monitored by TLC. The reaction mixture was then cooled by ice-water, filtrated, washed with water. Finally, purified by crystallization from a mixture of methylene chloride-petroleum ether.

\subsubsection{2-(2-Benzylidenehydrazinyl)-3-(phenylthio)quinoxaline (9a)}

Orange crystals, $0.5 \mathrm{~g}(90 \%)$ yield; m.p. $187-190{ }^{\circ} \mathrm{C}$. IR (KBr): $3358(\mathrm{NH}), 3049(=\mathrm{C}-\mathrm{H}), 1613(\mathrm{C}=\mathrm{N})$, and 748, 627 (C-S, asymmetric and symmetric stretching) $\mathrm{cm}^{-1} .{ }^{1} \mathrm{H}-\mathrm{NMR}\left(500 \mathrm{MHz}, \mathrm{DMSO}-d_{6}, 300 \mathrm{~K}\right)$ : 
$\delta 9.50$ (br. s, $1 \mathrm{H}, \mathrm{NH}, \mathrm{D}_{2} \mathrm{O}$ exchangeable), $8.65(\mathrm{~s}, 1 \mathrm{H}, \mathrm{CH}), 7.82-7.86(\mathrm{~m}, 2 \mathrm{H}, \mathrm{Ar}-\mathrm{H}), 7.67-7.60(\mathrm{~m}, 2 \mathrm{H}$, Ar-H), 7.48-7.38 (m, 6H, Ar-H), $7.37(\mathrm{~d}, 1 \mathrm{H}, J=8.0 \mathrm{~Hz}, \mathrm{Ar}-\mathrm{H}), 7.34-7.26(\mathrm{~m}, 1 \mathrm{H}, \mathrm{Ar}-\mathrm{H})$, and 7.05-7.14 (m, 1H, Ar-H) ppm. ${ }^{13} \mathrm{C}-\mathrm{NMR}$ (APT) (125 MHz, DMSO- $\left.d_{6}, 300 \mathrm{~K}\right): \delta 158.12$ (C), $156.52(\mathrm{CH}), 143.74$ (C), $135.47(\mathrm{CH}), 134.94(\mathrm{C}), 132.65(\mathrm{C}), 130.56(\mathrm{C}), 130.44(\mathrm{CH}), 129.22(\mathrm{CH}), 129.07(\mathrm{CH}), 128.66(\mathrm{CH})$, $128.61(\mathrm{CH}), 128.44(\mathrm{CH}), 128.41(\mathrm{C}), 126.80(\mathrm{CH}), 122.36(\mathrm{CH}), 115.18(\mathrm{CH}) \mathrm{ppm} . \mathrm{C}_{21} \mathrm{H}_{16} \mathrm{~N}_{4} \mathrm{~S}$ requires: C, $70.79 \%$; H, 4.49\%; N, 15.73\%. Found: C, 70.96\%; H, 4.61\%; N, $15.79 \%$.

\subsubsection{2-(2-(4-Nitrobenzylidene)hydrazinyl)-3-(phenylthio)quinoxaline (9b)}

Orange crystals (methylene chloride), $0.72 \mathrm{~g}(97 \%)$ yield; m.p. $200-202^{\circ} \mathrm{C}$. IR (KBr): $3344(\mathrm{NH})$, $3064(=\mathrm{C}-\mathrm{H}), 1599(\mathrm{C}=\mathrm{N}), 1547,1332\left(\mathrm{NO}_{2}\right.$, asymmetric and symmetric stretching), and 746, 626 (C-S, asymmetric and symmetric stretching) $\mathrm{cm}^{-1}$. ${ }^{1} \mathrm{H}-\mathrm{NMR}\left(500 \mathrm{MHz}, \mathrm{DMSO}-d_{6}, 300 \mathrm{~K}\right): \delta 8.90$ (brs, $1 \mathrm{H}, \mathrm{NH}, \mathrm{D}_{2} \mathrm{O}$ exchangeable), $8.72(\mathrm{~s}, 1 \mathrm{H}, \mathrm{CH}), 8.30(\mathrm{~d}, 2 \mathrm{H}, J=8.6 \mathrm{~Hz}, \mathrm{Ar}-\mathrm{H}), 7.98(\mathrm{~d}, 2 \mathrm{H}, J=8.8 \mathrm{~Hz}$, Ar-H), $7.63(\mathrm{~m}, 2 \mathrm{H}, \mathrm{Ar}-\mathrm{H}), 7.48-7.44(\mathrm{~m}, 3 \mathrm{H}, \mathrm{Ar}-\mathrm{H}), 7.38(\mathrm{~d}, 1 \mathrm{H}, J=8.0 \mathrm{~Hz}, \mathrm{Ar}-\mathrm{H}), 7.31(\mathrm{t}, 1 \mathrm{H}, J=7.4 \mathrm{~Hz}$, Ar-H), and 7.12 (d, $2 \mathrm{H}, J=7.6 \mathrm{~Hz}, \mathrm{Ar}-\mathrm{H}) \mathrm{ppm} .{ }^{13} \mathrm{C}-\mathrm{NMR}$ (APT) (125 MHz, DMSO- $\left.d_{6}, 300 \mathrm{~K}\right): \delta 157.90$ (C), $154.19(\mathrm{CH}), 147.98(\mathrm{C}), 144.51(\mathrm{C}), 141.22(\mathrm{C}), 135.44(\mathrm{CH}), 132.96(\mathrm{C}), 130.20(\mathrm{C}), 129.28(\mathrm{CH})$, $129.11(\mathrm{CH}), 129.10(\mathrm{C}), 128.65(\mathrm{CH}), 128.49(\mathrm{CH}), 127.05(\mathrm{CH}), 123.82(\mathrm{CH}), 122.86(\mathrm{CH}), 115.51(\mathrm{CH})$ ppm. $\mathrm{C}_{21} \mathrm{H}_{15} \mathrm{~N}_{5} \mathrm{SO}_{2}$ requires: $\mathrm{C}, 62.84 \% ; \mathrm{H}, 3.74 \% ; \mathrm{N}, 17.46 \%$. Found: $\mathrm{C}, 63.04 \% ; \mathrm{H}, 3.95 \% ; \mathrm{N}, 17.72 \%$.

\subsubsection{2-(2-(3-Nitrobenzylidene)hydrazinyl)-3-(phenylthio)quinoxaline (9c)}

Dark orange crystals (methanol-dimethylformamide), $0.71 \mathrm{~g}(96 \%)$ yield; m.p. $201-203^{\circ} \mathrm{C}$. IR (KBr): $3350(\mathrm{NH}), 3072(=\mathrm{C}-\mathrm{H}), 1617(\mathrm{C}=\mathrm{N}), 1522,1342\left(\mathrm{NO}_{2}\right.$, asymmetric and symmetric stretching), and 741, 654 (C-S, asymmetric and symmetric stretching) $\mathrm{cm}^{-1} .{ }^{1} \mathrm{H}-\mathrm{NMR}\left(500 \mathrm{MHz}, \mathrm{DMSO}-d_{6}, 300 \mathrm{~K}\right): \delta 8.88$ (brs, $1 \mathrm{H}, \mathrm{NH}, \mathrm{D}_{2} \mathrm{O}$ exchangeable), $8.70(\mathrm{~m}, 2 \mathrm{H}, \mathrm{CH}$, and Ar-H), $8.22(\mathrm{~d}, 1 \mathrm{H}, J=7.6 \mathrm{~Hz}, \mathrm{Ar}-\mathrm{H}), 8.10$ $(\mathrm{d}, 1 \mathrm{H}, J=7.2 \mathrm{~Hz}, \mathrm{Ar}-\mathrm{H}), 7.63(\mathrm{~m}, 2 \mathrm{H}, \mathrm{Ar}-\mathrm{H}), 7.57$ (t, 1H, J = 7.2 Hz, Ar-H), 7.50-7.44 (m, 3H, Ar-H), 7.43-7.29 (m, 3H, Ar-H), and 7.16 (t, $1 \mathrm{H}, J=4.7 \mathrm{~Hz}, \mathrm{Ar}-\mathrm{H})$ ppm. ${ }^{13} \mathrm{C}-\mathrm{NMR}$ (APT) (125 MHz, DMSO-d $d_{6}$, $300 \mathrm{~K}): \delta 157.92(\mathrm{C}), 154.36(\mathrm{CH}), 148.32(\mathrm{C}), 144.25(\mathrm{C}), 136.82(\mathrm{C}), 135.30(\mathrm{CH}), 134.36(\mathrm{CH}), 132.81(\mathrm{C})$, $130.32(\mathrm{C}), 130.13(\mathrm{CH}), 129.26(\mathrm{CH}), 129.10(\mathrm{CH}), 128.56(\mathrm{CH}), 128.54(\mathrm{C}), 127.02(\mathrm{CH}), 124.53(\mathrm{CH})$, $122.66(\mathrm{CH}), 122.42(\mathrm{CH}), 115.38(\mathrm{CH})$ ppm. $\mathrm{C}_{21} \mathrm{H}_{15} \mathrm{~N}_{5} \mathrm{SO}_{2}$ requires: $\mathrm{C}, 62.84 \% ; \mathrm{H}, 3.74 \% ; \mathrm{N}, 17.46 \%$. Found: $\mathrm{C}, 63.13 \% ; \mathrm{H}, 3.85 \%$; $\mathrm{N}, 17.67 \%$.

\subsubsection{2-(2-(4-Methoxybenzylidene)hydrazinyl)-3-(phenylthio)quinoxaline (9d)}

Dark orange crystals, 0.6 g (93\%) yield; m.p. 193-195 ${ }^{\circ} \mathrm{C}$. IR (KBr): 3359 (NH), $3053(=\mathrm{C}-\mathrm{H})$, $2963(-\mathrm{C}-\mathrm{H}), 1615(\mathrm{C}=\mathrm{N})$, and 744, 654 (C-S, asymmetric and symmetric stretching) $\mathrm{cm}^{-1} .{ }^{1} \mathrm{H}-\mathrm{NMR}$ $\left(500 \mathrm{MHz}, \mathrm{DMSO}-d_{6}, 300 \mathrm{~K}\right): \delta 8.60(\mathrm{~s}, 1 \mathrm{H}, \mathrm{CH}), 7.90(\mathrm{~s}, 1 \mathrm{H}, \mathrm{Ar}-\mathrm{H}), 7.68-7.59(\mathrm{~m}, 3 \mathrm{H}, \mathrm{Ar}-\mathrm{H}), 7.52-7.47$ $(\mathrm{m}, 2 \mathrm{H}, \mathrm{Ar}-\mathrm{H}), 7.42-7.46(\mathrm{~m}, 3 \mathrm{H}, \mathrm{Ar}-\mathrm{H}), 7.43-7.38(\mathrm{~m}, 1 \mathrm{H}, \mathrm{Ar}-\mathrm{H}), 7.34(\mathrm{t}, 1 \mathrm{H}, J=9.8 \mathrm{~Hz}, \mathrm{Ar}-\mathrm{H}), 7.11$ $(\mathrm{m}, 1 \mathrm{H}, \mathrm{Ar}-\mathrm{H}), 6.94(\mathrm{~m}, 1 \mathrm{H}, \mathrm{Ar}-\mathrm{H})$, and $3.85\left(\mathrm{~s}, 3 \mathrm{H}, \mathrm{CH}_{3}\right) \mathrm{ppm} .{ }^{13} \mathrm{C}-\mathrm{NMR}$ (APT) (125 MHz, DMSO- $d_{6}$, $300 \mathrm{~K})$ : $\delta 161.23(\mathrm{C}), 158.22(\mathrm{C}), 156.24(\mathrm{CH}), 143.30(\mathrm{C}), 135.46(\mathrm{CH}), 132.56(\mathrm{C}), 130.72(\mathrm{CH}), 130.13$ (C), $129.18(\mathrm{CH}), 129.06(\mathrm{CH}), 128.70(\mathrm{C}), 128.37(\mathrm{CH}), 127.60(\mathrm{C}), 126.91(\mathrm{CH}), 122.15(\mathrm{CH}), 115.27$ $(\mathrm{CH}), 114.14(\mathrm{CH}), 55.36\left(\mathrm{OCH}_{3}\right)$ ppm. $\mathrm{C}_{22} \mathrm{H}_{18} \mathrm{~N}_{4} \mathrm{~S}$ O requires: $\mathrm{C}, 68.39 \% ; \mathrm{H}, 4.66 \% ; \mathrm{N}, 14.51 \%$. Found: C: $67.98 ; \mathrm{H}: 4,79 ; \mathrm{N}: 14.32 \%$.

\subsubsection{2-(2-(4-Chlorobenzylidene)hydrazinyl)-3-(phenylthio)quinoxaline (9e)}

Dark orange crystals, 0.6 g (91\%) yield; m.p. 197-199 ${ }^{\circ} \mathrm{C}$. IR (KBr): 3355 (NH), 3050 (=C-H), 1615 $(\mathrm{C}=\mathrm{N})$, and 747, 637 (C-S, asymmetric and symmetric stretching) $\mathrm{cm}^{-1} .{ }^{1} \mathrm{H}-\mathrm{NMR}$ (500 MHz, DMSO- $d_{6}$, $300 \mathrm{~K}): \delta 8.65$ (s, 1H, CH), 7.74-7.78 (m, 2H, Ar-H), 7.68-7.58 (m, 3H, Ar-H), 7.48-7.44 (m, 3H, Ar-H), 7.42-7.27 (m, 4H, Ar-H), and $7.16(\mathrm{~m}, 1 \mathrm{H}, \mathrm{Ar}-\mathrm{H}) \mathrm{ppm} .{ }^{13} \mathrm{C}-\mathrm{NMR}(\mathrm{APT})\left(125 \mathrm{MHz}, \mathrm{CDCl}_{3}, 300 \mathrm{~K}\right)$ : $\delta 153.38(\mathrm{C}), 150.26(\mathrm{CH}), 138.96(\mathrm{C}), 130.50(\mathrm{CH}), 129.94(\mathrm{C}), 128.94(\mathrm{C}), 127.75(\mathrm{C}), 125.52(\mathrm{C}), 125.02$ $(\mathrm{CH}), 124.26(\mathrm{CH}), 124.13(\mathrm{CH}), 123.76(\mathrm{CH}), 123.66(\mathrm{C}), 123.53(\mathrm{CH}), 122.03(\mathrm{CH}), 117.50(\mathrm{CH}), 110.25$ (CH) ppm. $\mathrm{C}_{21} \mathrm{H}_{15} \mathrm{~N}_{4} \mathrm{~S} \mathrm{Cl}$ requires: C, $64.53 \% ; \mathrm{H}, 3.84 \%$;, $14.34 \%$. Found: C: $64.39 \%$; H: 3.97\%; $\mathrm{N}: 14.58 \%$. 
3.13.6. 4-((2-(3-(Phenylthio)quinoxalin-2-yl)hydrazono)methyl)phenol (9f)

Orange crystals, $0.6 \mathrm{~g}(90 \%)$ yield; m.p. $203-205^{\circ} \mathrm{C}$. IR (KBr): $3339(\mathrm{NH}), 3256(\mathrm{OH}), 3057(=\mathrm{C}-\mathrm{H})$, $1598(\mathrm{C}=\mathrm{N})$, and 748, 659 (C-S, asymmetric and symmetric stretching) $\mathrm{cm}^{-1} .{ }^{1} \mathrm{H}-\mathrm{NMR}(500 \mathrm{MHz}$, DMSO- $d_{6}, 300 \mathrm{~K}$ ): $\delta 11.01$ (brs, $1 \mathrm{H}, \mathrm{OH}, \mathrm{D}_{2} \mathrm{O}$ exchangeable), 10.00 (brs, $1 \mathrm{H}, \mathrm{NH}, \mathrm{D}_{2} \mathrm{O}$ exchangeable), $8.46(\mathrm{~s}, 1 \mathrm{H}, \mathrm{CH}), 7.87(\mathrm{~d}, 2 \mathrm{H}, J=8.0 \mathrm{~Hz}, \mathrm{Ar}-\mathrm{H}), 7.62-7.58(\mathrm{~m}, 2 \mathrm{H}, \mathrm{Ar}-\mathrm{H}), 7.52(\mathrm{~d}, 1 \mathrm{H}, J=8.1 \mathrm{~Hz}, \mathrm{Ar}-\mathrm{H})$, 7.50-7.45 (m, 3H, Ar-H), 7.28 (t, 1H, J = 7.4 Hz, Ar-H), $7.13(\mathrm{~d}, 1 \mathrm{H}, J=7.7 \mathrm{~Hz}, \mathrm{Ar}-\mathrm{H}), 7.00(\mathrm{t}, 1 \mathrm{H}$, $J=9.5 \mathrm{~Hz}, \mathrm{Ar}-\mathrm{H})$, and $6.86(\mathrm{~d}, 2 \mathrm{H}, J=8.6 \mathrm{~Hz}, \mathrm{Ar}-\mathrm{H}) \mathrm{ppm} .{ }^{13} \mathrm{C}-\mathrm{NMR}$ (APT) $\left(125 \mathrm{MHz}, \mathrm{DMSO}-d_{6}\right.$, $300 \mathrm{~K})$ : $\delta 159.83(\mathrm{C}), 158.23(\mathrm{C}), 156.52(\mathrm{CH}), 143.07(\mathrm{C}), 135.43(\mathrm{CH}), 132.48(\mathrm{C}), 130.76(\mathrm{C}), 130.29(\mathrm{CH})$, $129.13(\mathrm{CH}), 129.01(\mathrm{CH}), 128.69(\mathrm{C}), 128.30(\mathrm{CH}), 126.85(\mathrm{CH}), 126.02(\mathrm{C}), 122.02(\mathrm{CH}), 115.47(\mathrm{CH})$, $114.94(\mathrm{CH})$ ppm. $\mathrm{C}_{21} \mathrm{H}_{16} \mathrm{~N}_{4} \mathrm{SO}$ requires: $\mathrm{C}, 67.74 \% ; \mathrm{H}, 4.30 \%$; N, $15.05 \%$. Found: $\mathrm{C}, 67.93 \% ; \mathrm{H}, 4.43 \%$; $\mathrm{N}, 15.21 \%$.

\subsection{Synthesis of Benzimidazo[ $\left.2^{\prime}, 1^{\prime}: 2,3\right]$ thiazolo[4,5-b]quinoxaline (10)}

To a solution of 2-mercaptobenzimidazole $(1.13 \mathrm{~g}, 0.0075 \mathrm{~mol})$ and triethyl amine $(1.51 \mathrm{~g}, 0.015 \mathrm{~mol})$ in $15 \mathrm{~mL}$ methanol, a solution of 2,3-DCQ $1(0.5 \mathrm{~g}, 0.0025 \mathrm{~mol})$ in $15 \mathrm{~mL}$ methanol was added with constant stirring. Then the mixture was refluxed for $4 \mathrm{~h}$, monitored by TLC. The reaction mixture was then cooled by ice-water, filtered, washed with water, and crystalized from hot ethanol.

Colorless crystals, $0.65 \mathrm{~g}(94 \%)$ yield; m.p. $198-200^{\circ} \mathrm{C}$. IR (KBr): $3049(=\mathrm{C}-\mathrm{H})$ and $1565(\mathrm{C}=\mathrm{N}) \mathrm{cm}^{-1}$. ${ }^{1} \mathrm{H}-\mathrm{NMR}(500 \mathrm{MHz}, \mathrm{CDCl} 3,300 \mathrm{~K}): \delta 7.97(\mathrm{dd}, 1 \mathrm{H}, J=8.1,1.4 \mathrm{~Hz}, \mathrm{Ar}-\mathrm{H}), 7.85(\mathrm{dd}, 1 \mathrm{H}, J=8.2,1.4 \mathrm{~Hz}$, Ar-H), 7.69-7.57 (m, 4H, Ar-H), and $7.29(\mathrm{dd}, 2 \mathrm{H}, J=6.0,3.1 \mathrm{~Hz}, \mathrm{Ar}-\mathrm{H}) \mathrm{ppm} .{ }^{13} \mathrm{C}-\mathrm{NMR}(\mathrm{APT})(125 \mathrm{MHz}$, CDCl3, $300 \mathrm{~K}): \delta 153.29$ (C), 152.81 (C), 152.33 (C), 145.82 (C), 145.28 (C), 138.57 (C), 137.76 (C), 129.52 $(\mathrm{CH}), 127.40(\mathrm{CH}), 127.21(\mathrm{CH}), 126.79(\mathrm{CH}), 122.94(\mathrm{CH}), 122.54(\mathrm{CH}), 118.80(\mathrm{CH}), 110.61(\mathrm{CH}) \mathrm{ppm}$. C15H8N4S requires: C, 65.2\%; H, 2.89\%; N, 20.2\%. Found: C, 64.89\%; H, 3.04\%; N, 20.52\%.

${ }^{13} \mathrm{C}-\mathrm{NMR}$ and ${ }^{1} \mathrm{H}-\mathrm{NMR}$ spectrums are available in Supplementary Materials.

\section{Conclusions}

In summary, we described a simple and efficient synthetic method for 19 new quinoxalines with evaluation of their antimicrobial activity. The synthesized compounds were fully characterized using melting point, IR, ${ }^{1} \mathrm{H}-\mathrm{NMR},{ }^{13} \mathrm{C}-\mathrm{APT}-\mathrm{NMR}$, and elemental analysis. The tested compounds showed reasonable antibacterial activity against the strains of Staphylococcus aureus; Bacillus subtilis; Escherichia coli. Furthermore, among all the tested compounds, only $\mathbf{6 a}, \mathbf{6 b}$ and the pentacyclic compound 10 have considerable antifungal activity. The pentacyclic compound $\mathbf{1 0}$ exhibited the highest antifungal activity against both Candida albicans and Aspergillus flavus having a MIC value of $16 \mu \mathrm{g} / \mathrm{mL}$. On the other hand, compounds $\mathbf{2} \mathbf{d}$ and $\mathbf{3} \mathbf{c}$ showed the highest antibacterial activity against the Gram-negative bacterium Escherichia coli having MIC value of $8 \mu \mathrm{g} / \mathrm{mL}$. The results indicated that the sulfur functionality on quinoxaline may enhance its antifungal activity, while functionalization of quinoxaline with aromatic amines enhance its antibacterial activity. Further investigations of this aspect are in progress.

Supplementary Materials: Supplementary materials are available online.

Author Contributions: E.A.H., M.A.E.-A. and A.Z.O. designed the research; A.Z.O., M.A.E.-A. and M.A. performed the experimental work and analyzed the data; E.A.H., M.A.E.-A. and A.Z.O. wrote the manuscript; E.A.H., M.A.E.-A. and A.Z.O. revised the manuscript. All the authors discussed, edited, and approved the final version.

Funding: This research received no external funding.

Conflicts of Interest: Authors declare no conflicts of interest.

\section{References}

1. Kim, Y.B.; Kim, Y.H.; Park, J.Y.; Kim, S.K. Synthesis and biological activity of new quinoxaline antibiotics of echinomycin analogues. Bioorg. Med. Chem. Lett 2004, 14, 541-544. [CrossRef] [PubMed] 
2. Sakata, G.; Makino, K.; Kurasawa, Y. Recent progress in the quinoxaline chemistry. Synthesis and biological activity. Heterocycles 1988, 27, 2481-2515. [CrossRef]

3. Tsami, A.; Bünnagel, T.W.; Farrell, T.; Scharber, M.; Choulis, S.A.; Brabec, C.J.; Scherf, U. Alternating quinoxaline/oligothiophene copolymers—synthesis and unexpected absorption properties. J. Mater. Chem. 2007, 17, 1353-1355. [CrossRef]

4. Jaung, J.-Y. Synthesis and halochromism of new quinoxaline fluorescent dyes. Dyes Pigments 2006, 71, 245-250. [CrossRef]

5. Justin Thomas, K.; Velusamy, M.; Lin, J.T.; Chuen, C.-H.; Tao, Y.-T. Chromophore-labeled quinoxaline derivatives as efficient electroluminescent materials. Chem. Mater. 2005, 17, 1860-1866. [CrossRef]

6. Dailey, S.; Feast, W.J.; Peace, R.J.; Sage, I.C.; Till, S.; Wood, E.L. Synthesis and device characterisation of side-chain polymer electron transport materials for organic semiconductor applications. J. Mater. Chem. 2001, 11, 2238-2243. [CrossRef]

7. Kulkarni, A.P.; Zhu, Y.; Jenekhe, S.A. Quinoxaline-containing polyfluorenes: Synthesis, photophysics, and stable blue electroluminescence. Macromolecules 2005, 38, 1553-1563. [CrossRef]

8. Carta, A.; Loriga, M.; Piras, S.; Paglietti, G.; Colla, P.L.; Busonera, B.; Collu, G.; Loddo, R. Synthesis of variously substituted 3-phenoxymethyl quinoxalin-2-ones and quinoxalines capable to potentiate in vitro the antiproliferative activity of anticancer drugs in multi-drug resistant cell lines. Med. Chem. 2006, 2, 113-122.

9. Khan, S.A.; Mullick, P.; Pandit, S.; Kaushik, D. Synthesis of hydrazones derivatives of quinoxalinone-prospective antimicrobial and antiinflammatory agents. Acta Pol. Pharm. 2009, 66, 169-172.

10. Piras, S.; Loriga, M.; Paglietti, G. Quinoxaline chemistry. Part XVII. Methyl [4-(substituted 2-quinoxalinyloxy) phenyl] acetates and ethyl $N$-\{[4-(substituted 2-quinoxalinyloxy) phenyl] acetyl\} glutamates analogs of methotrexate: Synthesis and evaluation of in vitro anticancer activity. Farmaco 2004, 59, 185-194. [CrossRef]

11. El-Sabbagh, O.I.; El-Sadek, M.E.; Lashine, S.M.; Yassin, S.H.; El-Nabtity, S.M. Synthesis of new 2 (1H)-quinoxalinone derivatives for antimicrobial and antiinflammatory evaluation. Med. Chem. Res. 2009, 18, 782. [CrossRef]

12. Fonseca, T.; Gigante, B.; Marques, M.M.; Gilchrist, T.L.; De Clercq, E. Synthesis and antiviral evaluation of benzimidazoles, quinoxalines and indoles from dehydroabietic acid. Bioorg. Med. Chem 2004, 12, 103-112. [CrossRef] [PubMed]

13. Ali, I.; Al-Masoudi, I.; Hassan, H.G.; Al-Masoudi, N. Synthesis and anti-HIV activity of new homo acyclic nucleosides, 1-(pent-4-enyl) quinoxalin-2-ones and 2-(pent-4-enyloxy) quinoxalines. Chem Hetrocycl Com 2007, 43, 1052-1059. [CrossRef]

14. Gupta, D.; Ghosh, N.N.; Chandra, R. Synthesis and pharmacological evaluation of substituted 5-[4-[2-(6, 7-dimethyl-1, 2, 3, 4-tetrahydro-2-oxo-4-quinoxalinyl) ethoxy] phenyl] methylene] thiazolidine-2, 4-dione derivatives as potent euglycemic and hypolipidemic agents. Bioorg. Med. Chem. Lett. 2005, 15, 1019-1022. [CrossRef] [PubMed]

15. Yang, Y.; Zhang, S.; Wu, B.; Ma, M.; Chen, X.; Qin, X.; He, M.; Hussain, S.; Jing, C.; Ma, B. An efficient synthesis of quinoxalinone derivatives as potent inhibitors of aldose reductase. Chemmedchem 2012, 7 , 823-835. [CrossRef]

16. Sarges, R.; Howard, H.R.; Browne, R.G.; Lebel, L.A.; Seymour, P.A.; Koe, B.K. 4-Amino [1, 2, 4] triazolo [4, 3-a] quinoxalines. A novel class of potent adenosine receptor antagonists and potential rapid-onset antidepressants. J. Med. Chem. 1990, 33, 2240-2254. [CrossRef]

17. Ancizu, S.; Moreno, E.; Solano, B.; Villar, R.; Burguete, A.; Torres, E.; Pérez-Silanes, S.; Aldana, I.; Monge, A. New 3-methylquinoxaline-2-carboxamide 1, 4-di- $N$-oxide derivatives as anti-Mycobacterium tuberculosis agents. Biorg. Med. Chem. 2010, 18, 2713-2719. [CrossRef]

18. Aravind, K.; Ganesh, A.; Ashok, D. Microwave assisted synthesis, characterization and antibacterial activity of quinoxaline derivatives. J. Chem. Pharm. Res. 2013, 5, 48-52.

19. Abbas, H.-A.S.; Al-Marhabi, A.R.; Ammar, Y.A. Design, synthesis and biological evaluation of 2, 3-disubstituted and fused quinoxalines as potential anticancer and antimicrobial agents. Acta Pol. Pharm. 2017, 74, 445-458.

20. Hui, X.; Desrivot, J.; Bories, C.; Loiseau, P.M.; Franck, X.; Hocquemiller, R.; Figadere, B. Synthesis and antiprotozoal activity of some new synthetic substituted quinoxalines. Bioorg. Med. Chem. Lett. 2006, 16, 815-820. [CrossRef] 
21. Guillon, J.; Moreau, S.; Mouray, E.; Sinou, V.; Forfar, I.; Fabre, S.B.; Desplat, V.; Millet, P.; Parzy, D.; Jarry, C. New ferrocenic pyrrolo [1,2-a] quinoxaline derivatives: Synthesis, and in vitro antimalarial activity. Biorg. Med. Chem. 2008, 16, 9133-9144. [CrossRef] [PubMed]

22. Gao, H.; Yamasaki, E.F.; Chan, K.K.; Shen, L.L.; Snapka, R.M. Chloroquinoxaline sulfonamide (NSC 339004) is a topoisomerase II $\alpha / \beta$ poison. Cancer Res. 2000, 60, 5937-5940. [PubMed]

23. Gao, H.; Huang, K.-C.; Yamasaki, E.F.; Chan, K.K.; Chohan, L.; Snapka, R.M. XK469, a selective topoisomerase II $\beta$ poison. Proc. Natl. Acad. Sci. USA 1999, 96, 12168-12173. [CrossRef] [PubMed]

24. Gao, H. Characterization of cell lines resistant to topoisomerase targeting drugs and mechanistic studies of Quinoxaline solid tumor drugs-a new chemical class of topoisomerase II poisons. Ph.D. Thesis, The Ohio State University, Columbus, OH, USA, 2001.

25. Kakodkar, N.C.; Peddinti, R.; Kletzel, M.; Tian, Y.; Guerrero, L.J.; Undevia, S.D.; Geary, D.; Chlenski, A.; Yang, Q.; Salwen, H.R. The quinoxaline anti-tumor agent $R-(+)-X K 469$ inhibits neuroblastoma tumor growth. Pediatr. Blood Cancer 2011, 56, 164-167. [CrossRef] [PubMed]

26. Sagdic, O.; Tornuk, F. Antimicrobial properties of organosulfur compounds. In Dietary phytochemicals and microbes; Springer: Dordrecht, The Netherlands, 2012; pp. 127-156.

27. Wilson, E.A.; Demmig-Adams, B. Antioxidant, anti-inflammatory, and antimicrobial properties of garlic and onions. Nutr. Food Sci. 2007, 37, 178-183. [CrossRef]

28. Li, J.-L.; Zhao, W.; Zhou, C.; Zhang, Y.-X.; Li, H.-M.; Tang, Y.-L.; Liang, X.-H.; Chen, T.; Tang, Y.-J. Comparison of carbon-sulfur and carbon-amine bond in therapeutic drug: $4 \beta$-S-aromatic heterocyclic podophyllum derivatives display antitumor activity. Sci. Rep. 2015, 5, 14814-14830. [CrossRef]

29. Mishra, A.; Kumar, M.; Mishra, A.; Kumar, A.; Kant, R.; RS, T. Synthesis and antibacterial studies of some new amides of 5-sulphosalicylic acid. Neoplasma 1990, 41, 291-296.

30. Cho, C.S.; Ren, W.X. A recyclable copper catalysis in quinoxaline synthesis from $\alpha$-hydroxyketones and o-phenylenediamines. J. Organomet. Chem. 2009, 694, 3215-3217. [CrossRef]

31. Khaksar, S.; Rostamnezhad, F. A novel one-pot synthesis of quinoxaline derivatives in fluorinated alcohols. Bull. Korean Chem. Soc. 2012, 33, 2581-2584. [CrossRef]

32. Soleymani, R.; Niakan, N.; Tayeb, S.; Hakimi, S. Synthesis of novel aryl quinoxaline derivatives by new catalytic methods. Orient. J. Chem. 2012, 28, 687-701. [CrossRef]

33. Wang, L.; Liu, J.; Tian, H.; Qian, C. Ytterbium triflate-catalyzed heterocyclization of 1,2-phenylenediamines and alkyl oxalates under solvent-free conditions via Phillips reaction: A facile synthesis of quinoxaline-2,3-diones derivatives. Synth. Commun. 2004, 34, 1349-1357. [CrossRef]

34. Cogo, J.; Kaplum, V.; Sangi, D.P.; Ueda-Nakamura, T.; Corrêa, A.G.; Nakamura, C.V. Synthesis and biological evaluation of novel 2, 3-disubstituted quinoxaline derivatives as antileishmanial and antitrypanosomal agents. Eur. J. Med. Chem. 2015, 90, 107-123. [CrossRef] [PubMed]

35. Akar, D.; Incesu, Z.; Gündogdu-Karaburun, N.; Benkli, K.; Isikdag, I. Synthesis and antitumor activity of some 6-chloro- and 6,7-dichloro-2,3-disubstitutedquinoxaline derivatives. Turkish J. Pharm. Sci. 2004, 1, 193-202.

36. Gerlicz, A.; Kraska, J.; Wojciechowski, L. Quinoxaline derivatives. PL 83335A5, 31 December 1975.

37. Khan, S.A.; Mullick, P.; Manchanda, H. Synthesis and antimicrobial activity of 2,3-disubstituted quinoxalines. Indian J. Heterocycl. Chem. 2008, 18, 197-198.

38. Ajani, O.O.; Obafemi, C.A.; Nwinyi, O.C.; Akinpelu, D.A. Microwave assisted synthesis and antimicrobial activity of 2-quinoxalinone-3-hydrazone derivatives. Biorg. Med. Chem. 2010, 18, 214-221. [CrossRef] [PubMed]

39. Mickevičienè, K.; Baranauskaitè, R.; Kantminienė, K.; Stasevych, M.; Komarovska-Porokhnyavets, O.; Novikov, V. Synthesis and antimicrobial activity of $N$-substituted- $\beta$-amino acid derivatives containing 2-hydroxyphenyl, benzo [b] phenoxazine and quinoxaline moieties. Molecules 2015, 20, 3170-3189. [CrossRef] [PubMed]

40. Kumar, P.; Narasimhan, B. Hydrazides/hydrazones as antimicrobial and anticancer agents in the new millennium. Mini Rev. Med. Chem. 2013, 13, 971-987. [CrossRef]

41. Ge, B.; Bodeis, S.; Walker, R.D.; White, D.G.; Zhao, S.; McDermott, P.F.; Meng, J. Comparison of the Etest and agar dilution for in vitro antimicrobial susceptibility testing of Campylobacter. J. Antimicrob. Chemother. 2002, 50, 487-494. [CrossRef] 
42. Balouiri, M.; Sadiki, M.; Ibnsouda, S.K. Methods for in vitro evaluating antimicrobial activity: A review. J. Pharm. Anal. 2016, 6, 71-79. [CrossRef]

43. Pawar, P.Y.; Bhise, S.B. Synthesis and antibacterial activity of some new quinoxalinedione derivatives. Int. J. Chem. Sci. 2007, 5, 700-710.

44. Podsiadly, R. Synthesis and photochemical reaction of novel, visible-wavelength oxidizable polymerization sensitizer based on the $12 \mathrm{H}$-quinoxalino[2,3-b][1,4]benzothiazine skeleton. J. Photochem. Photobiol. A 2009, 202, 115-121. [CrossRef]

45. Lockhart, D.; Turner, E.E. Aryloxy derivatives of pyrimidines, quinoxalines and quinolines. J. Chem. Soc. 1937, 424-427. [CrossRef]

46. Carter, S.D.; Cheeseman, G.W.H. Quinoxalines and related compounds. X. The formation of indolo[2,3-b]quinoxalines and 2-p-aminophenyl-3-anilinoquinoxalines from 2-anilinoquinoxalines. Tetrahedron 1978, 34, 981-988. [CrossRef]

47. Hampel, O.; Rode, C.; Walther, D.; Beckert, R.; Gorls, H. New derivatives of quinoxaline - syntheses, complex formation and their application as controlling ligands for zinc catalyzed epoxide- $\mathrm{CO}_{2}$-copolymerization. Z. Naturforsch. B Chem. Sci. 2002, 57, 946-956. [CrossRef]

48. Ramalingam, P.; Ganapaty, S.; Babu, R.C.H. In vitro antimicrobial and antitubercular activity of some new hydrazides, hydrazones and sulfonamides of quinoxalines. J. Pharm. Chem. 2008, 2, 161-164.

Sample Availability: Samples of the compounds 2-10 are available from the authors. 